\title{
TOWARDS A FUNCTIONAL CALCULUS FOR SUBNORMAL TUPLES: THE MINIMAL NORMAL EXTENSION
}

\author{
JOHN B. CONWAY
}

\begin{abstract}
In this paper the study of a functional calculus for subnormal $n$ tuples is initiated and the minimal normal extension problem for this functional calculus is explored. This problem is shown to be equivalent to a mean approximation problem in several complex variables which is solved. An analogous uniform approximation problem is also explored. In addition these general results are applied together with The Area and the The Coarea Formula from Geometric Measure Theory to operators on Bergman spaces and to the tensor product of two subnormal operators. The minimal normal extension of the tensor product of the Bergman shift with itself is completely determined.
\end{abstract}

An $n$-tuple of commuting operators $\mathbf{S}=\left(S_{1}, \ldots, S_{n}\right)$ on a Hilbert space $\mathscr{H}$ is subnormal if there is an $n$-tuple $\mathbf{N}=\left(N_{1}, \ldots, N_{n}\right)$ of commuting normal operators on a Hilbert space $\mathscr{K}$ that contains $\mathscr{H}$ such that for $1 \leq j \leq n$, $N_{j} \mathscr{H} \subseteq \mathscr{H}$ and $S_{j}=N_{j} \mid \mathscr{H}$. For any commuting $n$-tuple of operators $\mathbf{S}$ there is a notion of spectrum, the Taylor spectrum of $\mathbf{S}$ [30] (also see [12]), denoted by $\sigma(\mathbf{S})$. This spectrum is a nonempty compact subset of $\mathbb{C}^{n}$. In this general theory it is possible to define $\phi(\mathbf{S})$ for any function $\phi$ analytic in a neighborhood of $\sigma(\mathbf{S})$. This functional calculus generalizes the usual Riesz functional calculus for a single operator. When $\mathbf{S}$ is assumed to be a subnormal $n$-tuple, however, a much richer functional calculus is possible. In this case $\phi(\mathbf{S})$ can be defined for functions $\phi$ that are weak* limits of analytic functions and $\phi(\mathbf{S})$ becomes a subnormal operator. The central question in this development is "What are the properties of this operator $\phi(\mathbf{S})$ and what are the relations between the operator and the function $\phi$ ?"

More generally, if $\mathbf{S}$ is an $n$-tuple and $\phi_{1}, \ldots, \phi_{q}$ are analytic functions defined in a neighborhood of the Taylor spectrum of $\mathbf{S}$, then $\phi(\mathbf{S}) \equiv\left(\phi_{1}(\mathbf{S}), \ldots\right.$, $\left.\phi_{q}(\mathbf{S})\right)$ is a commuting $q$-tuple of operators. If $\mathbf{S}$ is subnormal, then $\phi(\mathbf{S})$ can be defined for $\phi=\left(\phi_{1}, \ldots, \phi_{q}\right)$ consisting of functions that are weak* limits

Received by the editors January 31, 1989 and, in revised form, July 6, 1989.

1980 Mathematics Subject Classification (1985 Revision). Primary 47B20, 47A60; Secondary $32 \mathrm{~A} 35,47 \mathrm{~B} 38$.

The results here were delivered in a talk at the American Mathematical Society Summer Institute in Operator Theory held at the University of New Hampshire in July 1988.

During the preparation of this paper partial support was furnished by National Science Foundation grant DMS 87-00835. 
of analytic functions. What are the properties of this $q$-tuple $\phi(\mathbf{S})$ and what are the relations between this $q$-tuple of operators and the family of functions $\phi ?$

If $S$ is a single subnormal operator on a Hilbert space $\mathscr{H}$ and $N$ is its minimal normal extension acting on $\mathscr{K}$, then a functional calculus for $S$ was studied in [11] and further extensions were explored in [14, 15, 18]. These results illustrate an intimate connection between subnormal operator theory and analytic function theory. In a sense the present paper is an attempt to extend and explore the results of [11] and its progeny in the context of subnormal $n$ tuples. The difficulty in this enterprise is that, unlike the study of the functional calculus for a single operator, the required function theory is not available. In [11] heavy use was made of the work of Sarason [29] characterizing the weakstar closure of the polynomials in $L^{\infty}(\mu)$ for an arbitrary compactly supported measure on $\mathbb{C}$. The work of Chaumat [6] extending the results of Sarason to the case of rational functions was the core of the function theory used to extend [11]. Thus the development of the functional calculus for a single subnormal operator could be carried out on a landscape richly colored and sparkled by function theoretic gems. In the present situation, the answers to the requisite questions in several complex variables lie in dark shadows amidst the hueless multidimensional unknown.

In spite of the lack of function theory several results can be obtained for tuples. In particular, the minimal normal extension of $\phi(\mathbf{S})$ can be characterized. There is, however, a difficulty here in that this characterization is given in terms of the disintegration of a measure and such a disintegration is difficult to compute when given a fixed measure and function. Nevertheless for certain specific (and important) cases these computations can be performed. In particular, by using The Area Formula and The Coarea Formula, subnormal tuples on Hardy spaces and Bergman spaces can be given a rather complete discussion.

Computations aside, this result has theoretical importance and usefulness. In fact these methods were used in [9] to study single subnormal operators and recapture one of the main theorems of [11]. More recently this method was used [16] to give another proof of the main result of [18] as well as to give some small improvements.

The results in this paper were announced in [10].

This paper is not the first to study $n$-tuples of subnormal operators. In [31], Keren Yan showed that a subnormal $n$-tuple has a nontrivial invariant subspace. In [13 and 25] the spectral properties of subnormal tuples are studied.

This paper is organized as follows. In $\S 1$ some preliminary material is presented. In particular, the problem is stated precisely and its equivalence to an $L^{2}$ approximation problem is shown. In $\S 2$ an analogous uniform approximation problem is treated. Positive solutions to the uniform approximation problem give positive results for the operator theory problem, so this section is not a detour. It is also pointed out that this problem in uniform approximation 
is, in the special case of a polydisk, equivalent to questions about interpolating sets in the torus.

$\S 3$ contains the solution to the minimal normal extension problem (Theorem 3.5). The previous minimal normal extension results are limited to functions that are weak* limits in $L^{\infty}(\mu)$ of rational functions. The present results apply to bounded functions that are also $L^{2}(\mu)$ limits of rational functions.

$\S 4$ considers the subnormal $n$-tuple $\mathbf{S}$ defined by multiplication by the coordinate functions on the Bergman space $L_{a}^{2}(G)$ for a bounded open set $G$ in $\mathbb{C}^{n}$. With the aid of The Area Theorem and The Coarea Theorem the necessary disintegration is carried out. If $\phi=\left(\phi_{1}, \ldots, \phi_{q}\right)$ is a $q$-tuple of bounded analytic functions on $G$, it is shown (under some conditions) that $\phi(\mathbf{N})$ is the minimal normal extension of $\phi(\mathbf{S})$ if $q=n$ and it is not the minimal normal extension if $q<n$.

In $\S 5$ the results of this paper are applied to the study of the tensor product of two subnormal operators. For subnormal operators $S_{1}$ and $S_{2}$, the question is whether $N_{1} \otimes N_{2}$ is the minimal normal extension of $S_{1} \otimes S_{2}$; if not, what is the minimal normal extension of $S_{1} \otimes S_{2}$ ? As it turns out, it is rare that $N_{1} \otimes N_{2}$ is the minimal normal extension of $S_{1} \otimes S_{2}$. (It might be pointed out that there exists a paper in print that claims to show that $N_{1} \otimes N_{2}$ is always the minimal normal extension of $S_{1} \otimes S_{2}$.) For example, if $S$ is the Bergman shift, then $N \otimes N$ is not the minimal normal extension of $S \otimes S$; with the aid of $\S 4$, however, the minimal normal extension of $S \otimes S$ can be determined explicitly.

\section{Preliminaries}

Let $\mathbf{S}=\left(S_{1}, \ldots, S_{n}\right)$ be a subnormal $n$-tuple acting on a Hilbert space $\mathscr{H}$. Amongst all the normal extensions $\mathbf{N}=\left(N_{1}, \ldots, N_{n}\right)$ of $\mathbf{S}$ there are minimal ones, characterized by the requirement that $\mathscr{K}$, the space on which $\mathbf{N}$ acts, does not contain any proper subspace that contains $\mathscr{H}$ and simultaneously reduces each operator $N_{j}$. Just as in the case of a single subnormal operator, any two minimal normal extensions of a subnormal $n$-tuple are unitarily equivalent. For information on a single subnormal operator the reader can consult [7] and for subnormal $n$-tuples see [12]. For general information on operator theory see [8].

Note that under these conditions each operator $S_{j}$ is subnormal. It is known [22] that there are commuting subnormal operators $S_{1}$ and $S_{2}$ such that $\left(S_{1}, S_{2}\right)$ is not a subnormal pair. However there are necessary and sufficient conditions on an $n$-tuple of commuting subnormal operators for it to be a subnormal $n$-tuple (see, for example, $[3,20]$ ).

If $\mathbf{S}$ is a subnormal $n$-tuple with minimal normal extension $\mathbf{N}$, then $C^{*}(\mathbf{N})$, the $C^{*}$-algebra generated by $N_{1}, \ldots, N_{n}$, is an abelian $C^{*}$-algebra and is thus isometrically isomorphic to the algebra of continuous functions on its maximal ideal space. But since this algebra is generated by $N_{1}, \ldots, N_{n}$, its maximal ideal space is homeomorphic to a compact subset $X$ of $\mathbb{C}^{n}$ and $C^{*}(\mathbf{N})$ is 
isometrically isomorphic to $C(X)$ in such a way that the operator $N_{j}$ corresponds to the coordinate function $z_{j}$. The set $X$ is called the joint spectrum of $\mathbf{N}$. Also the von Neumann algebra generated by $N_{1}, \ldots, N_{n}, W^{*}(\mathbf{N})$, is an abelian von Neumann algebra and so it is isometrically isomorphic to $L^{\infty}(X, \mu)$, where $\mu$ is a regular Borel measure on $X$. Moreover, this isomorphism extends the previous one so that each $N_{j}$ corresponds to the coordinate function $z_{j}$. This isomorphism is, moreover, a weak* homeomorphism. If $\phi \in L^{\infty}(\mu)$, then the image of $\phi$ in $W^{*}(\mathbf{N})$ is denoted by $\phi(\mathbf{N})$. This map $\phi \rightarrow \phi(\mathbf{N})$ is the standard functional calculus for normal $n$-tuples.

Define

$$
\mathscr{R}(\mathbf{S})=\left\{\phi \in L^{\infty}(\mu): \phi(\mathbf{N}) \mathscr{H} \subseteq \mathscr{H}\right\} .
$$

The Taylor spectrum of $\mathbf{S}$ is a compact subset $Y$ of $\mathbb{C}^{n}$ and if $\phi$ is analytic in a neighborhood of $Y$, then $\phi \in \mathscr{R}=\mathscr{R}(\mathbf{S})$. This fact, as well as the relevant definitions, can be found in [12]. A knowledge of the Taylor spectrum is not needed here and the above result is only mentioned so as to establish that $\mathscr{R}$ contains many functions besides the polynomials. In fact, it is a result of Putinar [25] that $X \subseteq Y \subseteq \widehat{X}$, the polynomially convex hull of $X$. Therefore $P(X)$, the uniform closure of the polynomials in $C(X)$, is contained in $\mathscr{R}$.

1.1 Proposition. $\mathscr{R}(\mathbf{S})$ is a weak ${ }^{*}$ closed subalgebra of $L^{\infty}(\mu)$ and the map $\phi \rightarrow \phi(\mathbf{N}) \mid \mathscr{H}$ is an isometry and a weak ${ }^{*}$ homeomorphism of $\mathscr{R}(\mathbf{S})$ onto a weak ${ }^{*}$ closed subalgebra of the commutant of $\mathbf{S}$.

Proof. See p. 207 of [7].

Define $\mathscr{R}(\mathbf{S})^{(q)}$ to be the collection of all $q$-tuples of functions $\phi=\left(\phi_{1}, \ldots\right.$, $\left.\phi_{q}\right)$ with $\phi_{j}$ in $\mathscr{R}(\mathbf{S})$ for each $j$. For $\phi$ in $\mathscr{R}(\mathbf{S})^{(q)}$, define $\phi(\mathbf{S})$ to be the $q$-tuple $\left(\phi_{1}(\mathbf{S}), \ldots, \phi_{q}(\mathbf{S})\right) ; \phi(\mathbf{S})$ is a subnormal $q$-tuple with $\phi(\mathbf{N})=$ $\left(\phi_{1}(\mathbf{N}), \ldots, \phi_{q}(\mathbf{N})\right)$ as a normal extension. The central question addressed in this paper is "What is the minimal normal extension of $\phi(\mathbf{S})$ ?" In particular, is $\phi(\mathbf{N})$ the minimal normal extension of $\phi(\mathbf{S})$ ? The next result is a reformulation of Proposition 2.4 on p. 128 of [7]. The proof is analogous.

1.2 Proposition. If $\phi=\left(\phi_{1}, \ldots, \phi_{q}\right) \in \mathscr{R}(\mathbf{S})^{(q)}$, then the minimal normal extension of $\phi(\mathbf{S})$ is the restriction of $\phi(\mathbf{N})$ to the subspace

$$
\bigvee\left\{\phi_{1}(\mathbf{N})^{* n_{1}} \cdots \phi_{q}(\mathbf{N})^{* n_{q}} h: h \in \mathscr{H} \text { and } n_{j} \geq 0,1 \leq j \leq q\right\}
$$

The following example illustrates the connection with approximation problems in several complex variables.

1.3 Example. Let $X$ be a compact subset of $\mathbb{C}^{n}$ and let $\mu$ be a positive measure supported on $X$. Let $R^{2}(X, \mu)$ be the closure in $L^{2}(\mu)$ of the rational function with poles off $X$. For $1 \leq j \leq n$, let $S_{j}: R^{2}(X, \mu) \rightarrow R^{2}(X, \mu)$ be defined by $S_{j} f=z_{j} f$. Then $\mathbf{S}=\left(S_{1}, \ldots, S_{n}\right)$ is a subnormal $n$-tuple with 
minimal normal extension $\mathbf{N}=\left(N_{1}, \ldots, N_{n}\right)$ on $L^{2}(\mu)$, where $N_{j} f=z_{j} f$ for $f$ in $L^{2}(\mu)$. In fact, if $\mathscr{N}$ is the smallest reducing subspace for $N_{1}, \ldots, N_{n}$ that contains $R^{2}(X, \mu)$, then $\mathscr{N}$ must contain polynomials in $z_{1}, \ldots, z_{n}$ as well as their conjugates. By the Stone-Weierstrass Theorem, $\mathscr{N}=L^{2}(\mu)$. In this situation, $W^{*}(\mathbf{N})=L^{\infty}(\mu)$ and $\mathscr{R}(\mathbf{S})=L^{\infty}(\mu) \cap R^{2}(X, \mu)$. If $\phi \epsilon$ $\mathscr{R}(\mathbf{S})^{(q)}$, then the determination of the minimal normal extension of $\phi(\mathbf{S})$ is, by Proposition 1.2, equivalent to the approximation problem of determining the closed linear span in $L^{2}(\mu)$ of

$\left\{\bar{\phi}_{1}^{n_{1}} \ldots \bar{\phi}_{q}^{n_{q}} h: h\right.$ is a rational function with poles off

$$
\left.X \text { and } n_{j} \geq 0,1 \leq j \leq q\right\} .
$$

In particular, is this closed linear span equal to $L^{2}(\mu)$ ?

Another example related to the preceding one (and equivalent to it if $n=1$ ) is the following.

1.4 Example. Let $X$ be a compact subset of $\mathbb{C}^{n}$ and let $\mu$ be a positive measure supported on $X$. Let $A^{2}(X, \mu)$ be the closure in $L^{2}(\mu)$ of the functions analytic in a neighborhood of $X$. For $1 \leq j \leq n$, let $S_{j}: A^{2}(X, \mu) \rightarrow A^{2}(X, \mu)$ be defined by $S_{j} f=z_{j} f$. Then $\mathbf{S}=\left(S_{1}, \ldots, S_{n}\right)$ is a subnormal $n$-tuple with minimal normal extension $\mathbf{N}=\left(N_{1}, \ldots, N_{n}\right)$ on $L^{2}(\mu)$, where $N_{j} f=z_{j} f$ for $f$ in $L^{2}(\mu)$. Here $\mathscr{R}(\mathbf{S})=A^{2}(X, \mu) \cap L^{\infty}(\mu)$.

The next example, the tensor product of two subnormal operators, is an important special case.

1.5 Example. Let $T_{1}$ and $T_{2}$ be subnormal operators on $\mathscr{H}_{1}$ and $\mathscr{H}_{2}$ with minimal normal extensions $M_{1}$ and $M_{2}$ on $\mathscr{K}_{1}$ and $\mathscr{K}_{2}$. Let $\mathscr{H}=\mathscr{H}_{1} \otimes \mathscr{H}_{2}$, $S_{1}=T_{1} \otimes 1, S_{2}=1 \otimes T_{2}, \mathscr{K}=\mathscr{K}_{1} \otimes \mathscr{K}_{2}, N_{1}=M_{1} \otimes 1$, and $N_{2}=1 \otimes M_{2}$. Then $\left(N_{1}, N_{2}\right)$ is the minimal normal extension of the pair $\left(S_{1}, S_{2}\right)$. If $\phi\left(z_{1}, z_{2}\right)=$ $z_{1} z_{2}$, then $\phi\left(S_{1}, S_{2}\right)=T_{1} \otimes T_{2}$ and the above question, in this situation, becomes: What is the minimal normal extension of $T_{1} \otimes T_{2}$ and when is it equal to $M_{1} \otimes M_{2}$ ?

If, in Example 1.5, $T=T_{1}=T_{2}=$ the operator defined as multiplication by $z$ on $L_{a}^{2}(\mathbb{D})$, the analytic functions on the open unit disk $\mathbb{D}$ that are square integrable with respect to area measure, then the question is "What is the minimal normal extension of $T \otimes T$ ?" Indeed, this is the original question that prompted this work. Since $L_{a}^{2}(\mathbb{D}) \otimes L_{a}^{2}(\mathbb{D})=L_{a}^{2}\left(\mathbb{D}^{2}\right)$, this question is equivalent to finding the closed linear span of

$$
\left\{\left(\overline{z_{1} z_{2}}\right)^{n} z_{1}^{k} z_{2}^{m}: n, m, k \geq 0\right\}
$$

in $L^{2}\left(\mathbb{D}^{2}\right)$. This problem will be completely solved in $\S 5$ by using The Coarea Formula. In particular it will be seen that this closed linear span is not $L^{2}\left(\mathbb{D}^{2}\right)$. 


\section{UNIFORM APPROXIMATION}

In this section a type of uniform approximation problem in several complex variables is considered. Rather than consider the most general situation, we will restrict our attention to a particular case.

For a compact subset $X$ of $\mathbb{C}^{n}$ let $R(X)$ be the uniform closure of rational functions with poles off $X$. Fix compact subsets $X$ and $Y$ of $\mathbb{C}^{n}$ such that $X \subseteq Y$ and let $\phi \in R(Y)^{(q)}$ for some $q \geq 1$; let

$$
\mathscr{A}_{0}(X, Y ; \phi)=\operatorname{span}\left\{f \bar{\phi}_{1}^{n_{1}} \cdots \bar{\phi}_{q}^{n_{q}}: f \in R(Y) \text { and } n_{j} \geq 0 \text { for } 1 \leq j \leq q\right\}
$$

in $C(X)$, and let

$$
\mathscr{A}(X, Y ; \phi)=\text { the uniform closure of } \mathscr{A}_{0}(X, Y ; \phi)
$$

in $C(X)$. So $\mathscr{A}(X, Y ; \phi)$ is the smallest closed subalgebra of $C(X)$ that contains the rational functions with poles off $Y$ as well as the conjugates of $\phi_{1}, \ldots, \phi_{q}$. If $Y=X$, let $\mathscr{A}(X ; \phi)=\mathscr{A}(X, X ; \phi)$. A typical possibility for $X$ and $Y$ occurs when $X$ is arbitrary and $Y$ is the polynomially convex hull of $X, \hat{X}$.

A problem related to the central question of this paper is to determine $\mathscr{A}(X, Y ; \phi)$. In the case of a single variable this problem is essentially solved in [24].

The relation of this problem to the one asked in the introduction of this paper is established by taking $Y$ to be the Taylor spectrum of a subnormal $n$-tuple and $X$ to be the joint spectrum of its minimal normal extension. In fact, if $\mathscr{A}(X, Y ; \phi)=C(X)$, then positive information is obtained about the minimal normal extension problem as the next result illustrates.

2.1 Proposition. Let $\mathbf{S}$ be a subnormal $n$-tuple with minimal normal extension $\mathbf{N}$ and let $X$ and $Y$ be the Taylor spectrum of $\mathbf{N}$ and $\mathbf{S}$, respectively. If $\phi \in R(Y)^{(q)}$ and $\mathscr{A}(X, Y ; \phi)=C(X)$, then $\phi(\mathbf{N})$ is the minimal normal extension of $\phi(\mathbf{S})$.

Proof. Let $\mathscr{N}$ be as in Proposition 1.2. Clearly $\mathscr{N}$ is invariant for $\psi(\mathbf{N})$ for every $\psi$ in $\mathscr{A}(X, Y ; \phi)$. If $\mathscr{A}(X, Y ; \phi)=C(X)$, then $\bar{z}_{j} \in \mathscr{A}(X, Y ; \phi)$ and so $\mathscr{N}$ reduces $N_{j}$ for each $j$. Since $\mathbf{N}$ is the minimal normal extension of $\mathbf{S}, \mathscr{N}=\mathscr{K}$.

For a subalgebra $\mathscr{A}$ of $C(X)$, a subset $E$ of $X$ is a set of antisymmetry for $\mathscr{A}$ if the only functions in $\mathscr{A}$ that are real-valued on $E$ are those that are constant on $E$ (see [19 or 17, p. 60]). Let $\mathscr{E}$ be the collection of maximal sets of antisymmetry for $\mathscr{A}(X, Y ; \phi)$. The Bishop-Stone-Weierstrass Theorem [4] states that each set $E$ in $\mathscr{E}$ is a compact subset of $X, \mathscr{E}$ forms a partition of $X, \mathscr{A}(X, Y ; \phi) \mid E$ is closed in $C(E)$, and if $f \in C(X)$ such that $f \mid E \epsilon$ $\mathscr{A}(X, Y ; \phi) \mid E$ for every $E$ in $\mathscr{E}$, then $f \in \mathscr{A}(X, Y ; \phi)$. Also, if $F$ is a closed subset of $X$ which is the union of maximal sets of antisymmetry, then $\mathscr{A}(X, Y ; \phi) \mid F$ is closed in $C(F)[19$, Theorem 3.4].

For each $\zeta=\left(\zeta_{1}, \ldots, \zeta_{q}\right)$ in $\mathbb{C}^{q}$, let $X_{\zeta}=X \cap \phi^{-1}(\zeta)$. 
2.2 Lemma. If $E \in \mathscr{E}$, then there is a constant $\zeta$ in $\mathbb{C}^{q}$ such that $E \subseteq X_{\zeta}$. Thus each $X_{\zeta}$ is a closed set which is the union of maximal sets of antisymmetry. Proof. Since each $\phi_{j}$ and its conjugate belong to $\mathscr{A}(X, Y ; \phi)$, the real and imaginary parts of each $\phi_{j}$ are in $\mathscr{A}(X, Y ; \phi)$. Hence $\phi_{j}$ must be constant on each set of antisymmetry for $\mathscr{A}(X, Y ; \phi)$.

For each $\zeta$ in $\mathbb{C}^{q}$, let $R\left(X_{\zeta} ; Y\right)$ denote the uniform closure in $C\left(X_{\zeta}\right)$ of the rational functions with poles off $Y$. (If $X_{\zeta}$ is empty, then $R\left(X_{\zeta} ; Y\right)$ and $C\left(X_{\zeta}\right)$ are empty. $)$

2.3 Proposition. A function $f$ in $C(X)$ belongs to $\mathscr{A}(X, Y ; \phi)$ if and only if $f \mid X_{\zeta} \in R\left(X_{\zeta} ; Y\right)$ for every $\zeta$ in $\mathbb{C}^{q}$.

Proof. Because $\phi$ is constant on $X_{\zeta}, f \mid X_{\zeta} \in R\left(X_{\zeta} ; Y\right)$ for every $f$ in $\mathscr{A}_{0}(X, Y ; \phi)$; thus $\mathscr{A}(X, Y ; \phi) \mid X_{\zeta} \subseteq R\left(X_{\zeta} ; Y\right)$. But $\mathscr{A}(X, Y ; \phi) \mid X_{\zeta}$ is closed [19, Theorem 3.4]. Since $\mathscr{A}(X, Y ; \phi)$ clearly contains the rational functions with poles off $Y$, the reverse inclusion in valid. Hence $\mathscr{A}(X, Y ; \phi) \mid X_{\zeta}=$ $R\left(X_{\zeta} ; Y\right)$. Now let $f \in C(X)$ such that $f \mid X_{\zeta} \in R\left(X_{\zeta} ; Y\right)$ for every $\zeta$. Then $f\left|X_{\zeta} \in \mathscr{A}(X, Y ; \phi)\right| X_{\zeta}$. By Lemma $2.2, f|E \in \mathscr{A}(X, Y, \phi)| E$ for every maximal set of antisymmetry $E$. By the Bishop-Stone-Weierstrass Theorem, $f \in$ $\mathscr{A}(X, Y ; \phi)$.

2.4 Corollary. $\mathscr{A}(X, Y ; \phi)=C(X)$ if and only if $R\left(X_{\zeta} ; Y\right)=C\left(X_{\zeta}\right)$ for all $\zeta$ in $\mathbb{C}^{q}$.

The next result follows from Theorem 4.11 below. A proof will be given, however, in order to maintain the connectedness of the exposition. Moreover this proof is simpler than the proof of Theorem 4.8.

2.5 Theorem. If $X$ has a nonempty interior, $q<n$, and $\phi \in R(Y)^{(q)}$, then $\mathscr{A}(X, Y ; \phi) \neq C(X)$.

Proof. There is no loss in generality in assuming that each of the functions $\phi_{j}$ is not constant. Thus at least one partial derivative $\partial_{k} \phi_{i}$ is not identically 0 ; without loss of generality, it may be assumed that $\partial_{n} \phi_{q}$ is not identically 0 . Let $U$ be a polydisk with $\operatorname{cl} U \subseteq$ int $X$ such that $\left|\partial_{n} \phi_{q}\right| \geq \delta>0$ on $U$. Let $a \in U$ and put $\zeta=\phi_{q}(a)$. By the Implicit Function Theorem, there is a polydisk $W$ in $\mathbb{C}^{n-1}$, a disk $D$ in $\mathbb{C}$, and a function $f$ analytic in a neighborhood of $\operatorname{cl} W$ such that $a \in K \equiv \phi_{q}^{-1}(\zeta) \cap \operatorname{cl}(W \times D)=\{(w, f(w)): w \in \operatorname{cl} W\} \subseteq$ $U$. Since $K$ is contained in the polydisk $U, \widehat{K} \subseteq U \subseteq$ int $X$. Thus each function from $R(Y)$ can be approximated uniformly on $K$ by polynomials. Thus $\mathscr{A}_{0}(X, Y ; \phi) \mid K \subseteq \mathscr{A}(K, \widehat{K} ; \phi)=\mathscr{A}\left(K, \widehat{K} ;\left(\phi_{1}, \ldots, \phi_{q-1}\right)\right)$ since $\phi_{q}$ is constant on $K$.

If $\mathscr{A}(X, Y ; \phi)=C(X)$, then it follows that $\mathscr{A}\left(K, \widehat{K} ;\left(\phi_{1}, \ldots, \phi_{q-1}\right)\right)=$ $C(K)$. If $F(w)=(w, f(w))$ for $w$ in $\mathrm{cl} W$, this implies that

$$
\mathscr{A}\left(\mathrm{cl} W, \operatorname{cl} W ;\left(\phi_{1} \circ F, \ldots, \phi_{q-1} \circ F\right)\right)=C(\mathrm{cl} W) .
$$


The proof can now be completed by induction on the number of variables. If $n=2$ (the first case that makes sense), then $q=1$. So if $\mathscr{A}(X, Y ; \phi)=C(X)$, it follows that $C(\operatorname{cl}(W)=\mathscr{A}(\mathrm{cl} W, \operatorname{cl} W ; \varnothing)=P(\operatorname{cl} W)$, a contradiction. Now assume that the theorem is valid for some $n \geq 2$ and assume that $X \subseteq Y \subseteq$ $\mathbb{C}^{n+1}$. If $\mathscr{A}(X, Y ; \phi)=C(X)$, then

$$
C(\mathrm{cl} W)=\mathscr{A}\left(\mathrm{cl} W, \mathrm{cl} W ;\left(\phi_{1} \circ F, \ldots, \phi_{q-1} \circ F\right)\right)
$$

and $q-1<n$, contradicting the induction hypothesis.

If $q=n$, it does not necessarily follow that $\mathscr{A}(X, Y ; \phi)=C(X)$. The functions $\phi_{j}$ could fail to be linearly independent, for instance. More can go wrong. For example, let $X=Y=$ the closed polydisk in $\mathbb{C}^{2}$ and let $\phi_{1}$ and $\phi_{2}$ be the polynomials $\phi_{1}(z, w)=z w$ and $\phi_{2}(z, w)=z^{2} w$. Here $X_{0}=(\mathrm{cl} \mathbb{D} \times\{0\}) \cup(\{0\} \times \mathrm{cl} \mathbb{D})$ and clearly $R\left(X_{0} ; Y\right) \neq C\left(X_{0}\right)$. By Corollary 2.4, $\mathscr{A}(X, Y ; \phi) \neq C(X)$.

The exact determination of which $\phi$ in $R(Y)^{n}$ satisfy $\mathscr{A}(X, Y ; \phi)=C(X)$ seems difficult, even if $X=Y=$ a polydisk. Some sufficient conditions, however, can be given. For example, if $\phi$ is such that for every $\zeta$ in $\mathbb{C}^{n}$ the set $\phi^{-1}(\zeta)$ is a finite subset $X$, the $\mathscr{A}(X, Y ; \phi)=C(X)$.

What happens if int $X=\varnothing$ ? It may be that $\mathscr{A}(X, Y ; \phi)=C(X)$ even when $q<n$. The following is an example.

2.6 Proposition. Let $K$ be a compact subset of $\mathbb{C}$ such that $0 \notin K$, let $X=$ $\partial \mathbb{D} \times K$, and let $Y$ be the polynomially convex hull of $X$. If $\phi(z, w)=z w$, then $\mathscr{A}(X, Y ; \phi)=C(X)$.

Proof. Note that $0 \notin \phi(X)$ and for any $\zeta$ in $\phi(X), X_{\zeta}=\{(z, \zeta / z): z \in \partial \mathbb{D}$ and $z / \zeta \in K\}$. For fixed $\zeta$ and any polynomial $p(z, w), p(z, \zeta / z)$ is a rational function in $z$ whose only poles are at 0 and $\infty$. But such rational functions are dense in $C(\partial \mathbb{D})$ and so $R\left(X_{\zeta} ; Y\right)=C\left(X_{\zeta}\right)$ for every $\zeta$. By Corollary $2.4, \mathscr{A}(X, Y ; \phi)=C(X)$.

To simplify the final discussion of this section, assume that $X=(\partial \mathbb{D})^{n}$ and $Y=\mathrm{cl} \mathbb{D}^{n}$. If $\phi_{1}, \ldots, \phi_{q} \in A\left(\mathbb{D}^{n}\right)$, the polydisk algebra, then $\mathscr{A}(X, Y ; \phi)$ is the uniformly closed linear span in $C\left((\partial \mathbb{D})^{n}\right)$ of

$$
\left\{p \bar{\phi}_{1}^{n_{1}} \cdots \bar{\phi}_{q}^{n_{q}}: n_{j} \geq 0 \text { and } p \text { is a polynomial }\right\} \text {. }
$$

The level sets $X_{\zeta}$ are subsets of the distinguished boundary $X=(\partial \mathbb{D})^{n}$. Since each of the functions $\phi_{j}$ is constant on $X_{\zeta}$ and $\mathscr{A}(X, Y ; \phi) \mid X_{\zeta}$ is closed, we see that $\mathscr{A}(X, Y ; \phi)\left|X_{\zeta}=A\left(\mathbb{D}^{n}\right)\right| X_{\zeta}=P\left(X_{\zeta}\right)$. Hence the condition in Corollary 2.4 that $P\left(X_{\zeta}\right)=C\left(X_{\zeta}\right)$ is the condition that $X_{\zeta}$ is an interpolating set for the polydisk algebra (see [26] for the definitions). Conditions on a subset of the torus that guarantee that it is an interpolating set are unknown, aside from the generalities found in [26]. With extra assumptions about $\phi$, certain cone conditions can be given on $X_{\zeta}$ so that it is an interpolating set (see [28, p. 164] for further information). 
It is not hard to see that

$$
\mathscr{A}\left((\partial \mathbb{D})^{2}, \mathrm{cl} \mathbb{D}^{2} ; z w\right)=C\left((\partial \mathbb{D})^{2}\right)
$$

and

$$
\mathscr{A}\left((\partial \mathbb{D})^{2}, \mathrm{cl} \mathbb{D}^{2} ; z-w\right) \neq C\left((\partial \mathbb{D})^{2}\right)
$$

\section{THE MINIMAL NORMAL EXTENSION}

In this section the minimal normal extension of $\phi(\mathbf{S})$ is determined in full generality for any $\phi$ in $\mathscr{R}(\mathbf{S})^{(q)}$. The main tool for this is the disintegration of measures. The following version of this theorem will be used (see [1] or p. 58 of [5]).

3.1 Theorem. Suppose $X$ and $Z$ are compact metric spaces and $\mu$ is a positive regular Borel measure on $X$. If $\phi: X \rightarrow Z$ is a Borel map and $\nu=\mu \circ \phi^{-1}$, then there exists a unique mapping $\zeta \rightarrow \lambda_{\zeta}$ of $Z$ into $M(X)$ satisfying the following:

(a) $\lambda_{\zeta} \geq 0$ and $\left\|\lambda_{\zeta}\right\|=1$ for all $\zeta$ in $\phi(X)$.

(b) For $f$ in $C(X), \zeta \rightarrow \int_{Z} f d \lambda_{\zeta}$ is a $\nu$-integrable function on $Z$.

(c) $\lambda_{\zeta}$ is carried by $\phi^{-1}(\zeta)$ for all $\zeta$ in $Z$ (so $\lambda_{\zeta}=0$ if $\zeta \notin \phi(X)$ ).

(d) $\mu=\int \lambda_{\zeta} d \nu(\zeta)$.

(The meaning of $(\mathrm{d})$ is that for every $f$ in $C(X), \int f d \mu=\int\left[\int f d \lambda_{\zeta}\right] d \nu(\zeta)$.)

It is worth noting that the measure $\nu$ is also uniquely determined by the conditions (a) through (d). That is, if a measure $\kappa$ and a function $\zeta \rightarrow \eta_{\zeta}$ are given satisfying the above conditions, then $\kappa=\nu$ and $\lambda_{\zeta}=\eta_{\zeta}$ a.e. $\nu$.

With the notation of the preceding theorem, if $g \in L^{2}(\mu)$, then $\int|g|^{2} d \mu=$ $\int\left[\int_{\phi^{-1}(\zeta)}|g|^{2} \lambda_{\zeta}\right] d \nu(\zeta)$ (see [5, pp. 60-61]). With this in view, it is not difficult to show that $L^{2}(\mu)$ consists of all equivalence classes of Borel functions $g$ on $X$ such that for $[\nu]$ a.e. $\zeta, g \mid \phi^{-1}(\zeta) \in L^{2}\left(\lambda_{\zeta}\right)$ and $\zeta \rightarrow \int_{\phi^{-1}(\zeta)}|g|^{2} d \lambda_{\zeta}$ belongs to $L^{1}(\nu)$. This can be expressed using the language of direct integrals by the equation $L^{2}(\mu)=\int^{\oplus} L^{2}\left(\lambda_{\zeta}\right) d \nu(\zeta)$. It is possible to avoid this language and the theory of direct integrals will not be used here. Often, however, there are conceptual advantages in thinking in terms of direct integrals.

The notation used in the introduction and $\S 1$ will be temporarily abandoned here in that $X$ will not always denote the joint spectrum of $\mathbf{N}$ and $\mu$ will not always be its scalar-valued spectral measure. Instead, use the Spectral Theorem for commuting $n$-tuples of normal operators and modify standard results about single normal operators (for example, see [8, p. 279]) to obtain the following.

3.2 Theorem. If $\mathbf{N}=\left(N_{1}, \ldots, N_{n}\right)$ is a commuting $n$-tuple of normal operators on a separable Hilbert space, then there is a compact metric space $X$, bounded Borel functions $\chi_{1}, \ldots, \chi_{n}$ on $X$, and a regular Borel measure $\mu$ on $X$ such that each $N_{j}$ is unitarily equivalent to multiplication by $\chi_{j}$ on $L^{2}(\mu)$. 
In most of the applications here, $X$ will indeed be the joint spectrum of $\mathbf{N}$, the functions $\chi_{j}$ will be the coordinate functions $z_{j}$, and $\mu$ will be the scalar-valued spectral measure for $\mathbf{N}$. Using the notation of Theorem 3.2, if $\chi=\left(\chi_{1}, \ldots, \chi_{n}\right)$, then, in general, $\mu \circ \chi^{-1}$ is the scalar-valued spectral measure for $\mathbf{N}$ and the joint spectrum of $N$ is the $\mu$-essential range of $\chi$.

If $\mathbf{S}$ is a subnormal $n$-tuple with minimal normal extension $\mathbf{N}$ and $\mathbf{N}$ is represented as in the preceding theorem, then each $S_{j}$ is defined by multiplication by $\chi_{j}$ on some closed subspace $\mathscr{H}$ of $L^{2}(\mu)$ that is invariant under this multiplication. Some important facts are gathered together in the next theorem.

3.3 Theorem. Let $X$ be a compact metric space and $\mu$ a regular Borel measure on $X$, and let $\mathbf{N}$ denote the $n$-tuple of normal operators defined on $L^{2}(\mu)$ by multiplication by the bounded Borel functions $\chi_{j}, 1 \leq j \leq n$; put $\chi=$ $\left(\chi_{1}, \ldots, \chi_{n}\right)$. If $\mathscr{H}$ is an invariant subspace for $\mathbf{N}$ such that $\mathbf{N}$ is the minimal normal extension of $\mathbf{S} \equiv$ the restriction of $\mathbf{N}$ to $\mathscr{H}$, then

$\mathscr{R}(\mathbf{S})=\{\psi: \psi$ is a bounded Borel function on $Z$ and $(\psi \circ \chi) \mathscr{H} \subseteq \mathscr{H}\}$.

For this section fix the notation introduced in the preceding theorem. Also introduce the algebra

$$
\mathscr{B}(\mathbf{S})=\left\{\phi \in L^{\infty}(X, \mu): \phi \mathscr{H} \subseteq \mathscr{H}\right\} .
$$

Note that if $\psi \in \mathscr{R}(\mathbf{S})$, then $\psi \circ \chi \in \mathscr{B}(\mathbf{S})$, but that not every function in $\mathscr{B}(\mathbf{S})$ will arise in this way. Nevertheless, as mentioned before, all the applications given later in this paper will satisfy $\mathscr{B}(\mathbf{S})=\mathscr{R}(\mathbf{S})$.

Fix $\phi=\left(\phi_{1}, \ldots, \phi_{q}\right)$ in $\mathscr{B}(\mathbf{S})^{(q)}$ and let $Z=$ the $\mu$-essential range of $\phi$ in $\mathbb{C}^{q}$. Let $\mu=\int_{\phi^{-1}(\zeta)} \lambda_{\zeta} d \nu(\zeta)$ be the disintegration of $\mu$ with respect to $\phi$, where $\nu=\mu \circ \phi^{-1}$. It is useful to fix each $\phi_{j}$ as a specific bounded Borel function defined everywhere on $X$, rather that some $\mu$-equivalence class of functions. For each $\zeta$ in $Z$, let $X_{\zeta}=\phi^{-1}(\zeta)$ and for $f$ in $L^{2}(\mu)$ let $f_{\zeta}=f \mid X_{\zeta}$. (Once again a representative of $f$ in $L^{2}(\mu)$ is being chosen. It is pertinent to observe that it may be that each $X_{\zeta}$ has $\mu$ measure 0 , so that this representative of $f$ must be fixed. A consequence of the disintegration of measures is that even if each $X_{\zeta}$ has measure 0, the measure $\mu$ can be recaptured from the measures $\lambda_{\zeta}$ on $X_{\zeta}$. So, gentle reader, with the observation that this implies that $\lambda_{\zeta}$ is not the restriction of $\mu$ to $X_{\zeta}$, we will temper our measure theoretic comments and leave the implementation of these amenities to you.)

Now fix a countable dense subset $\mathscr{H}_{0}$ of $\mathscr{H}$; for convenience assume that $\mathscr{H}_{0}$ is a complex-rational manifold. Define

$$
\mathscr{K}_{\zeta}=\text { the } L^{2}\left(\lambda_{\zeta}\right) \text { closure of }\left\{h_{\zeta}: h \in \mathscr{H}_{0}\right\} \text {. }
$$

3.5 Theorem. If $\phi \in \mathscr{B}(\mathbf{S})^{(q)}$ and $\mathscr{K}_{\zeta}$ is defined as in (3.4), then the minimal normal extension of the subnormal $q$-tuple $\phi(\mathbf{S})$ is the q-tuple of normal 
operators defined by multiplication by the functions $\phi_{j}$ on the space

$$
\mathscr{K} \equiv\left\{f \in L^{2}(\mu): f_{\zeta} \in \mathscr{K}_{\zeta} \text { a.e. }[\nu]\right\} .
$$

Proof. By Proposition 1.2 it must be shown that the space $\mathscr{K}$ defined above is equal to the space

$$
\mathscr{N}=\bigvee\left\{\bar{\phi}_{1}^{n_{1}} \cdots \bar{\phi}_{q}^{n_{q}} h: h \in \mathscr{H}, 1 \leq j \leq q \text {, and } n_{j} \geq 0\right\} .
$$

First note that for each $j, \mathscr{K}$ reduces multiplication by $\phi_{j}$. In fact, if $f \in \mathscr{K}$ and $\zeta \in Z$ such that $f_{\zeta} \in \mathscr{K}_{\zeta}$, then the fact that $\phi_{j}=\zeta_{j}$ on $X_{\zeta}$, the support of $\lambda_{\zeta}$, implies that $\left(\phi_{j} f\right)_{\zeta}=\zeta_{j} f_{\zeta}$ and $\left(\bar{\phi}_{j} f\right)_{\zeta}=\bar{\zeta}_{j} f_{\zeta}$. Hence $\phi_{j} f$ and $\bar{\phi}_{j} f \in \mathscr{K}$ whenever $f \in \mathscr{K}$, and so $\mathscr{K}$ reduces each $\phi_{j}$. But $\mathscr{N}$ is the smallest subspace of $L^{2}(\mu)$ that contains $\mathscr{H}$ and reduces each $\phi_{j}$, so that $\mathscr{N} \subseteq \mathscr{K}$.

Now let $f \in \mathscr{K}$ such that $f \perp \mathscr{N}$. It will be shown that $f=0$. For $h$ in $\mathscr{H}$ and nonnegative integers $n_{1}, \ldots, n_{q}$, we have $\bar{\phi}_{q}^{n_{1}} \cdots \bar{\phi}_{q}^{n_{q}} h \in \mathscr{N}$ and so

$$
0=\left\langle\bar{\phi}_{1}^{n_{1}} \cdots \bar{\phi}_{q}^{n_{1}} h, f\right\rangle=\int\left\langle\left(\bar{\phi}_{1}^{n_{1}} \cdots \bar{\phi}_{q}^{n_{1}} h\right)_{\zeta}, f_{\zeta}\right\rangle_{\zeta} d \nu(\zeta),
$$

where $\langle\cdot, \cdot\rangle_{\zeta}$ is used to denote the inner product in $L^{2}\left(\lambda_{\zeta}\right)$. But each $\phi_{j}$ is constantly equal to $\zeta_{j}$ on the carrier of $\lambda_{\zeta}$. Thus this equation becomes

$$
0=\int \bar{\zeta}_{1}^{n_{1}} \cdots \bar{\zeta}_{q}^{n_{q}}\left\langle h_{\zeta}, f_{\zeta}\right\rangle_{\zeta} d \nu(\zeta)
$$

But $\mathscr{H}$ is invariant under multiplication by $\phi_{j}$ so that $h$ in this equation can be replaced by $\phi_{1}^{m_{1}} \cdots \phi_{q}^{m_{q}} h$ for any choice of $m_{1}, \ldots, m_{q}$. Doing this and again using the fact that each $\phi_{j}$ is constant of the carrier of $\lambda_{\zeta}$ we get

$$
0=\int \bar{\zeta}_{1}^{n_{1}} \cdots \bar{\zeta}_{q}^{n_{q}} \zeta_{1}^{m_{1}} \ldots \zeta_{q}^{m_{q}}\left\langle h_{\zeta}, f_{\zeta}\right\rangle d \nu(\zeta)
$$

This implies that for every $h$ in $\mathscr{H}$ and every polynomial $P$ in $\zeta_{1}, \ldots, \zeta_{q}$, $\bar{\zeta}_{1}, \ldots, \bar{\zeta}_{q}, 0=\int P(\zeta, \bar{\zeta})\left\langle h_{\zeta}, f_{\zeta}\right\rangle_{\zeta} d \nu(\zeta)$. By the Stone-Weierstrass Theorem, $\left\langle h_{\zeta}, f_{\zeta}\right\rangle_{\zeta}=0$ a.e. $[\nu]$. Since $\mathscr{H}_{0}$ is countable, this implies there is a Borel set $\Delta \subseteq \mathbb{C}^{q}$ with $\nu(\Delta)=0$ and such that $\left\langle h_{\zeta}, f_{\zeta}\right\rangle_{\zeta}=0$ for all $\zeta$ not in $\Delta$ and all $h$ in $\mathscr{H}_{0}$. But $f \in \mathscr{K}$ and this says that $f_{\zeta} \perp \mathscr{K}_{\zeta}$ for $\zeta$ not in $\Delta$. Thus $f_{\zeta}=0$ a.e. $[\nu]$ and so $f=0$.

3.6 Corollary. $\phi(\mathbf{N})$ is the minimal normal extension of $\phi(\mathbf{S})$ if and only if the space $\mathscr{K}_{\zeta}$ defined in (3.4) equals $L^{2}\left(\lambda_{\zeta}\right)$ a.e. $[\nu]$.

It is worthwhile interpreting this theorem and its corollary for special cases. Indeed, this is precisely the purpose of the remainder of this paper. Presently this is done for Example 1.3. 
3.7 Corollary. Let $X, \mu$, and $\phi$ be as in Example 1.3 and let $\mu=\int \lambda_{\zeta} d \nu(\zeta)$ be the disintegration of $\mu$ with respect to $\phi$. The minimal normal extension of the q-tuple of subnormal operators defined by multiplication by $\phi_{j}$ on $R^{2}(X, \mu)$ is the q-tuple of multiplication by $\phi_{j}$ on the space

$$
\left\{f \in L^{2}(\mu): f_{\zeta} \equiv f \mid \phi^{-1}(\zeta) \in R^{2}\left(X, \lambda_{\zeta}\right) \text { a.e. }[\nu]\right\} .
$$

3.8 Corollary. Let $X, \mu$, and $\phi$ be as in Example 1.3 and let $\mu=\int \lambda_{\zeta} d \nu(\zeta)$ be the disintegration of $\mu$ with respect to $\phi$. The minimal normal extension of multiplication by $\phi(\mathbf{S})$ on $R^{2}(X, \mu)$ is $\phi(\mathbf{N})$ on $L^{2}(\mu)$ if and only if $R^{2}\left(X, \lambda_{\zeta}\right)=L^{2}\left(\lambda_{\zeta}\right)$ a.e. $[\nu]$.

New information is gained for single subnormal operators from Theorem 3.5. Indeed, for any subnormal operator $S$, Theorem 3.5 determines the minimal normal extension of $\phi(S)$ for any function $\phi$ in the restriction algebra of $S$ (see [7] for the definition). We give the next corollary as an illustration. (The general case can be easily obtained by the interested reader after the appropriate notation is established.)

3.9 Corollary. If $\mu$ is a compactly supported measure on $\mathbb{C}, P^{2}(\mu)$ is the closure of the analytic polynomials in $L^{2}(\mu), S$ is defined as multiplication by $z$ on $P^{2}(\mu), \phi \in P^{2}(\mu) \cap L^{\infty}(\mu)$, and $\mu=\int \lambda_{\zeta} d \nu(\zeta)$ is the disintegration of $\mu$ with respect to $\phi$, then $M_{\phi}$ on $L^{2}(\mu)$ is the minimal normal extension of $\phi(S)$ if and only if $P^{2}\left(\lambda_{\zeta}\right)=L^{2}\left(\lambda_{\zeta}\right)$ a.e. $[\nu]$.

In the notation of Corollary 3.9, it was shown in [11] that if $\phi \in P^{\infty}(\mu)$, then the minimal normal extension of $M_{\phi}$ on $P^{2}(\mu)$ is $M_{\phi}$ on $L^{2}(\mu)$ provided $\phi$ is not constant on any component of the Sarason hull of $\mu$ (see [11 or 7] for the definitions). Corollary 3.9 says that the same conclusion holds if $\phi \in P^{2}(\mu) \cap L^{\infty}(\mu)$ provided $\phi$ is not "too constant"; that is, provided $\phi^{-1}(\zeta)$ is not very large for too many $\zeta$. A direct proof of the result for $\phi$ in $P^{\infty}(\mu)$ using the techniques of this paper can be found in [9]. This result from [11] has been extended in [18] and most recently the main result of [18] was given a proof using this technique of disintegration in [16].

\section{Operators on a Bergman space}

In this section Theorem 3.5 will be applied to the study of multiplication operators on the Bergman space $L_{a}^{2}(G)$, for $G$ a bounded open subset of $\mathbb{C}^{n}$. The main tools in this application are The Area Formula and The Coarea Formula. For the reader's convenience we state these results below in the form needed. A readable reference is [23].

To fix some notation, let $\mathscr{A}_{n}$ denote Lebesgue measure on $\mathbb{C}^{n}$. (Realize that this is $2 n$-dimensional Lebesgue measure. In what follows all dimensional statement are to be taken as complex dimensional statements.) If $G \subseteq \mathbb{C}^{n}$, then $L^{p}(G)$ denotes the $L^{p}$ space of the restriction of $\mathscr{A}_{n}$ to $G$. If $\phi: G \rightarrow \mathbb{C}^{q}$ is 
a smooth mapping and $q \leq n$, then $J_{q} \phi(a)$ is the $q$-dimensional Jacobian of $\phi$ at $a$ in $G$; this is defined as the maximum $q$-dimensional volume (that is, $q$-dimensional Hausdorff measure) of the image of a $q$-dimensional cube in $\mathbb{C}^{n}$ under the differential $D \phi(a)$ (see [23, p. 25]). $J_{q} \phi(a)^{2}$ turns out to be the sum of the squares of the determinants of the $(2 q \times 2 q)$ real submatrices of $D \phi(a)$, when $\phi$ is considered as a mapping from $\mathbb{R}^{2 n}$ into $\mathbb{R}^{2 q}$. In the case that $\phi$ is analytic (the case of interest to us), $D \phi(a)$ is the $q \times n$ complex matrix $\left[\partial_{k} \phi_{j}\right]$, where $\partial_{k}$ denotes the derivative with respect to $z_{k}$ and $\phi=\left(\phi_{1}, \ldots, \phi_{q}\right)$ (see [21, p. 42]). It follows (again see [21, p. 42]) that $J_{q} \phi(a)$ is the sum of the squares of the absolute values of all the $q \times q$ complex submatrices of $\left[\partial_{k} \phi_{j}\right]$. In particular, $J_{n} \phi(a)=\left|\operatorname{det}\left[\partial_{k} \phi_{j}\right]\right|^{2}$.

4.1 The Area Formula. If $G$ is an open subset of $\mathbb{C}^{n}$ and $\phi: G \rightarrow \mathbb{C}^{n}$ is a Lipschitz mapping, then for any $f$ in $L^{1}(G)$,

$$
\int_{G} f\left(J_{n} \phi\right) d \mathscr{A}_{n}(z)=\int_{\mathbb{C}^{n}} \sum\{f(z): \phi(z)=\zeta\} d \mathscr{A}_{n}(\zeta) .
$$

4.2 The Coarea Formula. If $G$ is an open subset of $\mathbb{C}^{n}, q<n$, and $\phi: G \rightarrow$ $\mathbb{C}^{q}$ is a Lipschitz mapping, then for any $f$ in $L^{1}(G)$,

$$
\int_{G} f\left(J_{q} \phi\right) d \mathscr{A}_{n}=\int_{\mathbb{C}^{q}}\left[\int_{\phi^{-1}(\zeta)} f(z) d H_{n-q}(z)\right] d \mathscr{A}_{q}(\zeta),
$$

where $H_{n-q}$ denotes $(n-q)$-dimensional Hausdorff measure. (Actually $(2 n-2 q)$-dimensional real Hausdorff measure.)

The two formulas stated here could be combined into one if 0-dimensional Hausdorff measure is taken to be counting measure.

The reader might be struck by the similarity of these two formulas with the disintegration of measures. Indeed, the uniqueness of the disintegration of a measure implies that these two formulas give the disintegration of $\mathscr{A}_{n} \mid G$ with respect to $\phi$, after suitable normalization. There is a difficulty here, however. It may be that the normalization cannot be carried out. For example, if $\phi: G \rightarrow \mathbb{C}^{n}$ and $J_{n} \phi$ is 0 on a set of positive $\mathscr{A}_{n}$ measure, then we will not be able to "divide" by $J_{n} \phi$ and carry out this normalization. This is, however, the only impediment. The proofs of the next two propositions are exercises in measure theory.

4.3 Proposition. If in $4.1 J_{n} \phi(z)>0$ a.e. $\left[\mathscr{A}_{n}\right]$, then for $f$ in $L^{1}(G)$

$$
\int_{G} f d \mathscr{A}_{n}=\int_{\mathbb{C}^{n}} \sum\left\{f(z) / J_{n} \phi(z): \phi(z)=\zeta\right\} d \mathscr{A}_{n}(\zeta) .
$$

4.4 Proposition. If in $4.2 J_{q} \phi(a)>0$ a.e. $\left[\mathscr{A}_{n}\right]$, then for $f$ in $L^{1}(G)$

$$
\int_{G} f d \mathscr{A}_{n}=\int_{\mathbb{C}^{q}}\left[\int_{\phi^{-1}(\zeta)} f(z) / J_{q} \phi(z) d H_{n-q}(z)\right] d \mathscr{A}_{q}(\zeta) .
$$


With this done, the disintegration of $\mathscr{A}_{n}$ with respect to $\phi$ can be found. The next proposition is a consequence of the uniqueness of the disintegration of a measure.

4.5 Proposition. If $G$ is an open subset of $\mathbb{C}^{n}$ and $\phi: G \rightarrow \mathbb{C}^{n}$ is a Lipschitz mapping such that $J_{n} \phi>0$ a.e. $\left[\mathscr{A}_{n}\right]$ on $G$, then the disintegration of $\mathscr{A}_{n} \mid G$ with respect to $\phi$ is given by

$$
\mathscr{A}_{n} \mid G=\int \lambda_{\zeta} d \nu(\zeta)
$$

where $d \nu(\zeta)=\Lambda(\zeta) d \mathscr{A}_{n}(\zeta)$ with $\Lambda(\zeta)=\sum\left\{\left[J_{n} \phi(z)\right]^{-1}: \phi(z)=\zeta\right\}$ and $d \lambda_{\zeta}(z)$ $=\Lambda(\zeta)^{-1} \sum\left\{\left[J_{n} \phi(z)\right]^{-1} \delta_{z}: \phi(z)=\zeta\right\}$.

4.6 Proposition. If $G$ is an open subset of $\mathbb{C}^{n}, q<n$, and $\phi: G \rightarrow \mathbb{C}^{q}$ is a Lipschitz mapping such that $J_{q} \phi>0$ a.e. $\left[\mathscr{A}_{n}\right]$ on $G$, then the disintegration of $\mathscr{A}_{n} \mid G$ with respect to $\phi$ is given by

$$
\mathscr{A}_{n} \mid G=\int \lambda_{\zeta} d \nu(\zeta)
$$

where $d \nu(\zeta)=\Lambda(\zeta) d \mathscr{A}_{q}(\zeta)$ with $\Lambda(\zeta)=\int_{\phi^{-1}(\zeta)}\left[J_{q} \phi(z)\right]^{-1} d H_{n-q}(z)$ and $d \lambda_{\zeta}(z)=\Lambda(\zeta)^{-1}\left[J_{q} \phi(z)\right]^{-1} d H_{n-q}(z)$ on $\phi^{-1}(\zeta)$.

One of the principal results of this section can now be presented. To state this result, another function theoretic concept must be introduced.

4.7 Definition. If $G$ is a bounded open subset of $\mathbb{C}^{n}$ and $\left\{a_{j}\right\}$ is a discrete sequence in $G$, then $\left\{a_{j}\right\}$ is said to be weakly interpolating if $\left\{\left\{f\left(a_{j}\right)\right\}: f \in\right.$ $\left.H^{\infty}(G)\right\}$ is weak ${ }^{*}$ dense in $l^{\infty}$.

A sufficient condition for a sequence $\left\{a_{j}\right\}$ to be weakly interpolating is that for each $i \geq 1$ there is a function $f$ in $H^{\infty}(G)$ such that $f\left(a_{i}\right)=1$ and $f\left(a_{j}\right)=0$ for $j \neq i$. Since we are assuming that $G$ is bounded, this implies that all finite sequences in $G$ are weakly interpolating. In fact, for any finite subset $\left\{a_{1}, \ldots, a_{m}\right\}$ of $G,\left\{\left(f\left(a_{1}\right), \ldots, f\left(a_{m}\right)\right): f \in H^{\infty}(G)\right\}=\mathbb{C}^{m}$.

If $n=1$, every zero sequence of a bounded analytic function on $G$ is weakly interpolating. For $n \geq 2$ there seems, at first, to be a paucity of candidates for weakly interpolating sequences. The next lemma is pertinent to this paper and at least suggests one place to look for weakly interpolating sequences.

4.8 Lemma. If $G$ is an open subset of $\mathbb{C}^{n}$ and $\phi: G \rightarrow \mathbb{C}^{n}$ is an analytic mapping, then there is a subset $\Delta$ of $\mathbb{C}^{n}$ with $\mathscr{A}_{n}(\Delta)=0$ and such that for every $\zeta$ not in $\Delta, \phi^{-1}(\zeta)$ is a discrete sequence in $G$.

Proof. Let $G=\bigcup_{j=1}^{\infty} K_{j}$, where, for each $j \geq 1, K_{j}$ is compact and $K_{j} \subseteq$ $K_{j+1}$. Since $\phi$ is analytic, $\phi \mid$ int $K_{j}$ is Lipschitz. Hence the Area Theorem implies $\infty>\int J_{n} \phi d \mathscr{A}_{n}=\int_{\mathbb{C}^{n}} \#\left(\phi^{-1}(\zeta) \cap \operatorname{int} K_{j}\right) d \mathscr{A}_{n}(\zeta)$, where for any set $E$, $\#(E)$ is the number of points in $E$ when $E$ is a finite set, and $\#(E)=\infty$ if $E$ 
is infinite. Thus $\#\left(\phi^{-1}(\zeta) \cap\right.$ int $\left.K_{j}\right)<\infty$ a.e. $\left[\mathscr{A}_{n}\right]$ and so there is a measurable set $\Delta_{j}$ with $\mathscr{A}_{n}\left(\Delta_{j}\right)=0$ and such that $\#\left(\phi^{-1}(\zeta) \cap\right.$ int $\left.K_{j}\right)<\infty$ for $\zeta \notin \Delta_{j}$. If $\Delta=\bigcup_{j=1}^{\infty} \Delta_{j}$, the lemma is proved.

The next theorem is one of the main results of this paper. The preceding lemma says the hypothesis of this theorem is not outlandish. In fact, it may be that this hypothesis is always satisfied; I know of no example in which it is not. After the theorem we will see a sufficient condition for the hypothesis to be fulfilled.

4.9 Theorem. Let $G$ be a bounded open subset of $\mathbb{C}^{n}$, let $\phi_{1}, \ldots, \phi_{n} \in$ $H^{\infty}(G)$, and define $\phi=\left(\phi_{1}, \ldots, \phi_{n}\right): G \rightarrow \mathbb{C}^{n}$; assume that $J_{n} \phi$ is not constantly 0 on any component of $G$. If there is a measurable set $\Delta$ contained in $\mathbb{C}^{n}$ with $\mathscr{A}_{n}(\Delta)=0$ such that for $\zeta \notin \Delta, \phi^{-1}(\zeta)$ is a weakly interpolating sequence, then the minimal normal extension of $\left(M_{\phi_{1}}, \ldots, M_{\phi_{n}}\right)$ acting on $L_{a}^{2}(G)$ is $\left(M_{\phi_{1}}, \ldots, M_{\phi_{n}}\right)$ acting on $L^{2}(G)$.

Proof. First observe that $J_{n} \phi>0$ a.e. $\left[\mathscr{A}_{n}\right]$. In fact, the function $z \rightarrow$ $\operatorname{det}\left[\partial_{k} \phi_{j}(z)\right]$ is analytic on $G$ and not identically 0 an any component of $G$. By 14.4 .9 of [27], $J_{n} \phi>0$ a.e. $\left[\mathscr{A}_{n}\right]$. Thus the disintegration of $\mathscr{A}_{n} \mid G$ is given by Proposition 4.5. Let $\lambda_{\zeta}$ and $\nu$ be as in 4.5.

By hypothesis, there is a measurable set $\Delta$ with $\mathscr{A}_{n}(\Delta)=0$ such that $\phi^{-1}(\zeta)$ is a weakly interpolating sequence when $\zeta \notin \Delta$. But if $\zeta \notin \Delta, \lambda_{\zeta}$ and counting measure on $\phi^{-1}(\zeta)$ are mutually absolutely continuous. Thus $L^{\infty}\left(\lambda_{\zeta}\right)$ and $l^{\infty}$ are naturally isomorphic and weak* homeomorphic. Since $\phi^{-1}(\zeta)$ is weakly interpolating if $\zeta \notin \Delta, H^{\infty}(G) \mid \phi^{-1}(\zeta)$ is weak* dense in $L^{\infty}\left(\lambda_{\zeta}\right)$. Using the notation (3.4), this implies $\mathscr{K}_{\zeta}=L^{2}\left(\lambda_{\zeta}\right)$ if $\zeta \notin \Delta$. By Corollary 3.6, the theorem follows.

As stated before the theorem, I have no example in which the hypothesis of Theorem 4.9 is not satisfied. In light of Lemma 4.8, this question may be phrased as follows. If $\phi: G \rightarrow \mathbb{C}^{n}$ is an analytic mapping, $\zeta \in \mathbb{C}^{n}$, and $\phi^{-1}(\zeta)$ is a discrete sequence, must it be that $\phi^{-1}(\zeta)$ is weakly interpolating?

Here is a sufficient condition for the hypothesis of the preceding theorem to be satisfied.

4.10 Corollary. Let $G$ and $\phi$ be as in Theorem 4.9 and in addition assume that $\int_{G} J_{n} \phi d \mathscr{A}_{n}<\infty$. Then the minimal normal extension of $\left(M_{\phi_{1}}, \ldots, M_{\phi_{n}}\right)$ acting on $L_{a}^{2}(G)$ is $\left(M_{\phi_{1}}, \ldots, M_{\phi_{n}}\right)$ acting on $L^{2}(G)$.

Proof. Using The Area Formula and the hypothesis, we get that

$$
\int \#\left(\phi^{-1}(\zeta)\right) d \mathscr{A}_{n}(\zeta)<\infty \text {. }
$$

Hence there is a set $\Delta$ with $\mathscr{A}_{n}(\Delta)=0$ and $\#\left(\phi^{-1}(\zeta)\right)<\infty$ for $\zeta \notin \Delta$. The corollary is now immediate from the theorem. 
The use of the hypothesis $\int_{G} J_{n} \phi d \mathscr{A}_{n}<\infty$ seems somewhat simplistic. It is used to conclude that $\#\left(\phi^{-1}(\zeta)\right)<\infty$ a.e. and hence $P^{2}(\mu)=L^{2}(\mu)$ for any measure $\mu$ supported on $\phi^{-1}(\zeta)$. Surely this is overkill, but there does not seem to be a way at present to get around this without further advances in function theory. Indeed, if $F$ is an analytic variety in $\mathbb{C}^{n}$ such that $P^{2}(\mu)=L^{2}(\mu)$ for every compactly supported measure $\mu$ carried by $F$, then it is easy to see that $P(K)=C(K)$ for every compact subset $K$ of $F$. Conditions on $F$ that this happen do not seem to exist.

The Coarea Formula will now be used to prove the following theorem.

4.11 Theorem. If $G$ is a bounded open subset of $\mathbb{C}^{n}, q<n$, and $\phi_{1}, \ldots, \phi_{q} \in$ $H^{\infty}(G)$, then the closed linear span of $\left\{\bar{\phi}_{1}^{n_{1}} \cdots \bar{\phi}_{q}^{n_{q}} h: h \in L_{a}^{2}(G), n_{j} \geq 0,1 \leq\right.$ $j \leq q$ \} is not equal to $L^{2}(G)$.

A lemma must be established before proving the theorem.

4.12 Lemma. Let $G$ be a bounded open subset of $\mathbb{C}^{n}, \phi \in H^{\infty}(G), \zeta \in$ $\phi(G)$, and assume there is an open subset $W$ of $\mathbb{C}^{n-1}$ and an analytic function $f: W \rightarrow \mathbb{C}$ such that $\phi^{-1}(\zeta)=\{(w, f(w)): w \in W\}$. If $\partial_{n} \phi(w, f(w)) \neq 0$ a.e. $\left[\mathscr{A}_{n-1}\right]$ on $W, H_{n-1}$ is $(n-1)$-dimensional Hausdorff measure on $\phi^{-1}(\zeta)$, and $h \in L^{1}\left(H_{n-1}\right)$, then

$$
\int_{\phi^{-1}(\zeta)} h d H_{n-1}=\int_{W} \frac{h(w, f(w))}{\left|\partial_{n} \phi(w, f(w))\right|^{2}} \sum_{k=1}^{n}\left|\partial_{k} \phi(w, f(w))\right|^{2} d \mathscr{A}_{n-1}(w) .
$$

Proof. Using the equation $\zeta=\phi(w, f(w))$, differentiation gives that for $1 \leq$ $j<n$,

$$
\partial_{n} \phi(w, f(w)) \frac{\partial f}{\partial w_{j}}=-\partial_{j} \phi(w, f(w)) .
$$

But $\partial_{n} \phi(w, f(w)) \neq 0$ a.e. $\left[\mathscr{A}_{n-1}\right]$ on $W$ by hypothesis. Hence

$$
\frac{\partial f}{\partial w_{j}}=-\frac{\partial_{j} \phi(w, f(w))}{\partial_{n} \phi(w, f(w))}
$$

But $\phi^{-1}(\zeta)$ is a $(2 n-2)$ real manifold and so Hausdorff measure on $\phi^{-1}(\zeta)$ is surface measure. Thus for $h$ in $L^{1}\left(\phi^{-1}(\zeta), H_{n-1}\right)$,

$$
\int_{\phi^{-1}(\zeta)} h d H_{n-1}=\int_{W} h(w, f(w))\left|J_{n} F(w)\right| d \mathscr{A}_{n-1}(w),
$$

where $F(w)=(w, f(w))$. Since $F$ is analytic,

$$
\begin{aligned}
\left|J_{n} F\right| & =1+\sum_{j=1}^{n-1}\left|\frac{\partial f}{\partial w_{j}}\right|^{2}=1+\sum_{j=1}^{n-1}\left|\frac{\partial_{j} \phi(w, f(w))}{\partial_{n} \phi(w, f(w))}\right|^{2} \\
& =\left|\partial_{n} \phi(w, f(w))\right|^{-2} \sum_{j=1}^{n}\left|\partial_{j} \phi(w, f(w))\right|^{2} .
\end{aligned}
$$

The lemma now follows. 
Proof of Theorem 4.11. Note that the case $n=1$ does not make sense and so we will assume that $n \geq 2$.

Let $\mathscr{K}=\bigvee\left\{\bar{\phi}_{1}^{n_{1}} \cdots \bar{\phi}_{q}^{n_{q}} h: h \in L_{a}^{2}(G), 1 \leq j \leq q\right.$, and $\left.n_{j} \geq 0\right\}$ in $L^{2}(G)$. If $\partial_{n} \phi_{j} \equiv 0$ for all $j, 1 \leq j \leq q$, then clearly $\mathscr{K} \neq L^{2}(G)$ as each function in $\mathscr{K}$ would then be analytic in the variable $z_{n}$. Thus it can be assumed, by renumbering if necessary, that $\partial_{n} \phi_{q}$ is not identically 0 on $G$. Therefore there is a polydisk $D$ and constants $\delta$ and $M$ such that $\operatorname{cl} D \subseteq G$ and $0<\delta \leq$ $\left|\partial_{n} \phi_{q}\right| \leq M<\infty$ on $D$.

Let $\mathscr{M}$ be the closed linear span in $L^{2}(D)$ of $\left\{\bar{\phi}_{1}^{n_{1}} \cdots \bar{\phi}_{q}^{n_{q}} h: h \in L_{a}^{2}(G)\right.$, $1 \leq j \leq q$, and $\left.n_{j} \geq 0\right\}$. Suppose the conclusion of the theorem is false and $\mathscr{K}=L^{2}(G)$. It follows that $\mathscr{M}=L^{2}(D)$.

Since $D$ is a polydisk whose closure is contained in $G$, it must be that $\mathscr{M}$, and hence $L^{2}(D)$, is the closed linear span $\left\{\bar{\phi}_{1}^{n_{1}} \cdots \bar{\phi}_{q}^{n_{q}} p: p\right.$ is a polynomial and $n_{j} \geq 0$ for $\left.1 \leq j \leq q\right\}$. Let $\mu=\mathscr{A}_{n} \mid D$. By Proposition 4.6 the disintegration of $\mu$ with respect to $\phi_{q}$ is given by $\mu=\int \lambda_{\zeta} d \nu(\zeta)$, where $\lambda_{\zeta}=\left[J_{1} \phi_{q}\right]^{-1} H_{n-1}$ on $\phi_{q}^{-1}(\zeta)$ (except for a normalizing constant), $J_{1} \phi_{q}=\sum_{k}\left|\partial_{k} \phi_{q}\right|^{2}$, and $\nu$ is the restriction of $\mathscr{A}_{1}$ to $Z=\phi_{q}(D)$. (Since the measure $\left[J_{1} \phi_{q}\right]^{-1} H_{n-1}$ is a nonzero scalar multiple of the actual $\lambda_{\zeta}$, the $L^{2}$ closure of any set yields the same set of functions for either measure; thus we can safely assume that $\lambda_{\zeta}=$ $\left[J_{1} \phi_{q}\right]^{-1} H_{n-1}$.) For $\zeta$ in $Z$, let $\mathscr{M}_{\zeta}=$ the closed linear span in $L^{2}\left(\lambda_{\zeta}\right)$ of the polynomials with complex rational coefficients. Since $\mathscr{M}=L^{2}(D)$, Corollary 3.6 implies that $\mathscr{M}_{\zeta}=L^{2}\left(\lambda_{\zeta}\right)$ a.e. $[\nu]$.

Now fix any point $a$ in $D$ and let $\zeta=\phi_{q}(a)$; assume that $a$ was chosen so that $\mathscr{M}_{\zeta}=L^{2}\left(\lambda_{\zeta}\right)$. The Complex Implicit Function Theorem implies there is a polydisk $W$ in $\mathbb{C}^{n-1}$, a disk $\Delta$ in $\mathbb{C}$, and a function $f$ analytic in a neighborhood of $\mathrm{cl} W$ such that $a \in \phi_{q}^{-1}(\zeta) \cap(W \times \Delta)=\{(w, f(w)): w \in W\}$. By the preceding lemma, if $h \in L^{1}\left(\lambda_{\zeta} \mid W \times \Delta\right)$, then

$$
\begin{aligned}
\int h d \lambda_{\zeta} & =\int_{\phi_{q}^{-1}(\zeta)} h\left|J_{1} \phi_{q}\right|^{-1} d H_{n-1} \\
& =\int_{W} h(w, f(w))\left|\partial_{n} \phi_{q}(w, f(w))\right|^{-2} d \mathscr{A}_{n-1} .
\end{aligned}
$$

Hence the transformation $h \rightarrow h(w, f(w))$ defines an isometry of $L^{2}\left(W \times \Delta, \lambda_{\zeta}\right)$ onto $L^{2}\left(W,\left|\partial_{n} \phi_{q}(w, f(w))\right|^{-2} \mathscr{A}_{n-1}\right)$. Put $F(w)=(w, f(w))$; the fact that $\mathscr{M}_{\zeta}=L^{2}\left(\lambda_{\zeta}\right)$ implies that $L^{2}\left(W,\left|\partial_{n} \phi_{q}(w, f(w))\right|^{-2} \mathscr{A}_{n-1}\right)$ is the closed linear span of $\{p \circ F: p$ is a polynomial with complex rational coefficients $\}$. Now for any polynomial $p$,

$$
(p \circ F)(w)=p\left(w_{1}, \ldots, w_{n-1}, f\left(w_{1}, \ldots, w_{n-1}\right)\right)
$$

is analytic in a neighborhood of $\operatorname{cl} W$ since $f$ is. Thus $p \circ F$ can be approximated uniformly on $W$ by polynomials. This, combined with the fact that 
$\left|\partial_{n} \phi_{q}(w, f(w))\right|^{-2}$ is bounded above and below on $W$, implies that

$$
L^{2}\left(W, \mathscr{A}_{n-1}\right)=P^{2}\left(\mathscr{A}_{n-1} \mid W\right) .
$$

But this is a contradiction since $P^{2}\left(\mathscr{A}_{n-1} \mid W\right)=L_{a}^{2}(W)$ for any polydisk $W$ in $\mathbb{C}^{n-1}$.

\section{THE TENSOR PRODUCT OF TWO SUBNORMAL OPERATORS}

In this section the tensor product of two subnormal operators is studied. If $S_{1}$ and $S_{2}$ are subnormal operators with minimal normal extensions $N_{1}$ and $N_{2}$, what is the minimal normal extension of $S_{1} \otimes S_{2}$ ? Is it $N_{1} \otimes N_{2}$ ? Theorem 3.5 can be used to answer this question and this can then be applied to certain specific classes of examples such as operators on Bergman spaces. Also, the results of $\S 2$ on uniform approximation can be applied to the study of the tensor product of the operator multiplication by $z$ on the Hardy space of certain regions in $\mathbb{C}$ with an arbitrary subnormal operator. In this latter case the number 0 plays a crucial role.

The first result will dramatically illustrate the importance of 0 to this problem.

5.1 Proposition. Suppose $S_{1}$ and $S_{2}$ are subnormal operators with minimal normal extension $N_{1}$ and $N_{2}$. If $S_{1}$ is not normal and $N_{2}$ has a nontrivial kernel, then $N_{1} \otimes N_{2}$ is not the minimal normal extension of $S_{1} \otimes S_{2}$.

Proof. By hypothesis, $\mathscr{H}_{1}^{\perp} \otimes \operatorname{ker} N_{2}$ is a nontrivial subspace of $\operatorname{ker}\left(N_{1} \otimes N_{2}\right)$; clearly this space is also orthogonal to $\mathscr{H}_{1} \otimes \mathscr{H}_{2}$. Hence the restriction of $N_{1} \otimes N_{2}$ to $\left[\mathscr{H}_{1}^{\perp} \otimes\left(\operatorname{ker} N_{2}\right)\right]^{\perp}$ is a normal extension of $S_{1} \otimes S_{2}$.

Fix the following notation for the remainder of this section. Represent the subnormal operators $S_{1}$ and $S_{2}$ as multiplication by $\chi_{i}$ on the closed subspace $\mathscr{H}_{i}$ of $L^{2}\left(X_{i}, \mu_{i}\right), i=1,2$, where $N_{i}=M_{\chi_{i}}$ on $L^{2}\left(X_{i}, \mu_{i}\right)$ is the minimal normal extension of $S_{i}$. Note that $L^{2}\left(X_{1}, \mu_{1}\right) \otimes L^{2}\left(X_{2}, \mu_{2}\right)=L^{2}(X, \mu)$, where $X=X_{1} \times X_{2}$ and $\mu=\mu_{1} \times \mu_{2}$. (If $h_{i}: X_{i} \rightarrow \mathbb{C}$, a notational convenience is to let $h_{1} \otimes h_{2}$ denote the function from $X$ into $\mathbb{C}$ defined by $\left(h_{1} \otimes h_{2}\right)\left(x_{1}, x_{2}\right)=$ $h_{1}\left(x_{1}\right) h_{2}\left(x_{2}\right)$.) Let $\mathscr{H}=\mathscr{H}_{1} \otimes \mathscr{H}_{2}$; that is, $\mathscr{H}$ is the closure in $L^{2}(X, \mu)$ of $\left\{h_{1} \otimes h_{2}: h_{i} \in \mathscr{H}_{i}\right\}$. Also if $\phi: X \rightarrow \mathbb{C}$ is defined by $\phi=\chi_{1} \otimes \chi_{2}$, then $S_{1} \otimes S_{2}=M_{\phi}$ on $\mathscr{H}$ and $N_{1} \otimes N_{2}=M_{\phi}$ on $L^{2}(X, \mu)$. As in Example 1.5, $\left(N_{1} \otimes 1,1 \otimes N_{2}\right)$ is the minimal normal extension of the pair $\left(S_{1} \otimes 1,1 \otimes S_{2}\right)$. Let $\mu=\int \lambda_{\zeta} d \nu(\zeta)$ be the disintegration of $\mu$ with respect to $\phi$, define $\mathscr{K}_{\zeta}$ as in (3.4), and let

$$
\mathscr{K}=\left\{f \in L^{2}(X, \mu): f \mid \phi^{-1}(\zeta) \in \mathscr{K}_{\zeta} \text { a.e. }[\nu]\right\} .
$$

The following result is an immediate consequence of Theorem 3.5.

5.2 Theorem. With the notation of the preceding paragraph, the minimal normal extension of $S_{1} \otimes S_{2}$ is multiplication by the function $\chi_{1} \otimes \chi_{2}$ on the space $\mathscr{K}$. 
Now assume that $N_{1}$ and $N_{2}$ have no kernels. Thus $\mu_{i}\left(\chi_{i}^{-1}(\{0\})\right)=0$. It follows that $\nu(\{0\})=0$ and so it suffices to consider $X_{\zeta}=\phi^{-1}(\zeta)$ only for $\zeta \neq 0$. Note that if $\left(x_{1}, x_{2}\right) \in X_{\zeta}$ and $\zeta \neq 0$, then $\chi_{1}\left(x_{1}\right) \chi_{2}\left(x_{2}\right)=\zeta$ and so $\chi_{2}\left(x_{2}\right)=\zeta / \chi_{1}\left(x_{1}\right)$.

5.3 Theorem. Let $S_{1}$ be a subnormal operator such that $\sigma\left(N_{1}\right) \subseteq \partial \sigma\left(S_{1}\right)$ and assume that int $\sigma\left(S_{1}\right)$ is connected, $0 \in \operatorname{int} \sigma\left(S_{1}\right)$, and $R\left(\partial \sigma\left(S_{1}\right)\right)=C\left(\partial \sigma\left(S_{1}\right)\right)$. If $S_{2}$ is any subnormal operator such that $\operatorname{ker} N_{2}=(0)$, then $N_{1} \otimes N_{2}$ is the minimal normal extension of $S_{1} \otimes S_{2}$.

Proof. Again, maintain the notation fixed above and let $K=\sigma\left(S_{1}\right)$. If $r$ is a rational function with poles off $K$ and $p$ is any analytic polynomial of one variable, then $\left(r \circ \chi_{1}\right) \otimes\left(p \circ \chi_{2}\right) \mathscr{H} \subseteq \mathscr{H}$ and so $r\left(\chi_{1}\right) p\left(\chi_{2}\right) \mathscr{K}_{\zeta} \subseteq \mathscr{K}_{\zeta}$. (Actually the set $\mathscr{H}_{0}$ must be carefully chosen so that $r\left(\chi_{1}\right) p\left(\chi_{2}\right) \mathscr{H}_{0} \subseteq \mathscr{H}_{0}$ when $r$ and $p$ have rational coefficients. As stated before, these measure-theoretic points are left to the reader.) But on $X_{\zeta}$,

$$
(r \otimes p)\left(\chi_{1}\left(x_{1}\right) \chi_{2}\left(x_{2}\right)\right)=r\left(\chi_{1}\left(x_{1}\right)\right) p\left(\zeta / \chi_{1}\left(x_{1}\right)\right)=f\left(\chi_{1}\left(x_{1}\right)\right)
$$

for a rational function $f$ whose poles lie in $\{0\} \cup\{\mathbb{C} \backslash K\}$. Note that if $f$ is any such rational function, then $f\left(\chi_{1}\left(x_{1}\right)\right)=r\left(\chi_{1}\left(x_{1}\right)\right) p\left(\zeta / \chi_{1}\left(x_{1}\right)\right)$ for an appropriate choice of $r$ and $p$. Therefore if $\zeta \neq 0,\left(f\left(\chi_{1}\right) \otimes 1\right) \mathscr{K}_{\zeta} \subseteq \mathscr{K}_{\zeta}$ for every rational function $f$ whose poles lie in $\{0\} \cup\{\mathbb{C} \backslash K\}$.

But the set of rational functions with poles in $\{0\} \cup\{\mathbb{C} \backslash K\}$ are dense in $C\left(\sigma\left(N_{1}\right)\right)$ by hypothesis. Thus $\left(f\left(\chi_{1}\right) \otimes 1\right) \mathscr{K}_{\zeta} \subseteq \mathscr{K}_{\zeta}$ for every continuous $f$ on $\sigma\left(N_{1}\right)$ and $\zeta \neq 0$. In particular, $\left(\bar{\chi}_{1} \otimes 1\right) \mathscr{K}_{\zeta} \subseteq \mathscr{K}_{\zeta}$ for $\zeta \neq 0$. Since $\mathscr{K}$ is always invariant for $M_{\chi_{1}} \otimes 1=N_{1} \otimes 1$, this implies that $\mathscr{K}$ reduces $N_{1} \otimes 1$. But also $\left(\bar{\chi}_{1}^{-1} \otimes 1\right) \mathscr{K}_{\zeta} \subseteq \mathscr{K}_{\zeta}$ for $\zeta \neq 0$ and since $\mathscr{K}$ reduces $N_{1} \otimes N_{2}, \mathscr{K}$ must be invariant for $1 \otimes N_{2}^{*}=\left(N_{1}^{-1 *} \otimes 1\right)\left(N_{1}^{*} \otimes N_{2}^{*}\right)$. Thus $\mathscr{K}$ is reducing for both $N_{1} \otimes 1$ and $1 \otimes N_{2}$. Since $\left(N_{1} \otimes 1,1 \otimes N_{2}\right)$ is the minimal normal extension of the pair $\left(S_{1} \otimes 1,1 \otimes S_{2}\right)$, this implies that $\mathscr{K}=L^{2}(\mu)$.

5.4 Corollary. If $S_{1}$ is the unilateral shift of any multiplicity and $S_{2}$ is any subnormal operator whose minimal normal extension has no kernel, then $N_{1} \otimes N_{2}$ is the minimal normal extension of $S_{1} \otimes S_{2}$.

For the remainder of this section, attention will be restricted to subnormal operators whose minimal normal extension is cyclic; call such operators fundamental. For fundamental subnormal operators a more convenient representation of the minimal normal extension of the tensor product can be obtained. If $S$ is a fundamental subnormal operator, then $N$ can be represented as multiplication by $z$ on $L^{2}(\mu)$ for some compactly supported measure $\mu$ on $\mathbb{C}$ and $S$ is represented as multiplication by $z$ on a closed subspace $\mathscr{H}$ of $L^{2}(\mu)$ such that $z \mathscr{H} \subseteq \mathscr{H}$. Moreover, this representation can be arranged so that $1 \in \mathscr{H}$. (This last fact is not so obvious; see [7, Exercise 7, p. 383].) Call this a preferred representation of $S$. Note that for the preferred representation, $\mathscr{H}$ 
contains all polynomials in $z$. Later our attention will be further restricted to the case where the subnormal operators are the natural ones on Bergman spaces and Hardy spaces.

So assume that $\mu_{1}$ and $\mu_{2}$ are compactly supported measures on $\mathbb{C}$ for $i=1,2$, let $\mathscr{H}_{i}$ be a closed subspace of $L^{2}\left(\mu_{i}\right)$ such that $z \mathscr{H}_{i} \subseteq \mathscr{H}_{i}$ and $1 \in \mathscr{H}_{i} ; N_{i} f=z f$ for $f$ in $L^{2}\left(\mu_{i}\right)$ and $S_{i} h=z h$ for $h$ in $\mathscr{H}_{i}$. In light of Proposition 5.1, it will be assumed throughout that

$$
\mu_{1}(\{0\})=\mu_{2}(\{0\})=0 .
$$

Retaining the notation introduced in the paragraph preceding Theorem 5.2, we now have that $X_{i} \subseteq \mathbb{C}$ and $\chi_{i}\left(z_{i}\right)=z_{i}, i=1,2$. Also $\phi\left(z_{1}, z_{2}\right)=z_{1} z_{2}$. For $\zeta \neq 0, \phi^{-1}(\zeta)=\left\{\left(z_{1}, z_{2}\right): z_{1} z_{2}=\zeta\right\}=\left\{(z, \zeta / z): z \in X_{1}\right.$ and $\zeta / z \in$ $\left.X_{2}\right\}$. Let $Y_{\zeta}=\left\{z \in X_{1}: \zeta / z \in X_{2}\right\}$. Note that $Y_{\zeta}$ is homeomorphic to $\phi^{-1}(\zeta)$ under the map $z \rightarrow(z, \zeta / z)$. Accordingly, there is a measure $\eta_{\zeta}$ on $Y_{\zeta}$ such that for every $f$ in $C\left(\phi^{-1}(\zeta)\right)$,

$$
\int_{\phi^{-1}(\zeta)} f d \lambda_{\zeta}=\int_{Y_{\zeta}} f(z, \zeta / z) d \eta_{\zeta} .
$$

From (5.6) we have that the map $h \rightarrow h(z, \zeta / z)$ defines a unitary operator from $\mathscr{K}_{\zeta}$ onto

$$
\mathscr{N}_{\zeta}=\bigvee\left\{h(z, \zeta / z): h \in \mathscr{H}_{1} \otimes \mathscr{H}_{2}\right\}
$$

in $L^{2}\left(\eta_{\zeta}\right)$. Applying Theorem 3.5 yields the following.

5.8 Theorem. If $S_{1}$ and $S_{2}$ are fundamental subnormal operators and their preferred representations satisfy (5.5), then the minimal normal extension of $S_{1} \otimes S_{2}$ is multiplication by $z_{1} z_{2}$ on the subspace

$$
\mathscr{N} \equiv\left\{f \in L^{2}(\mu): f(z, \zeta / z) \in \mathscr{N}_{\zeta} \text { a.e. }[\nu]\right\} .
$$

There may be a conceptual advantage here in using the language of direct integrals. Theorem 5.8 can be rephrased by saying that the minimal normal extension of $S_{1} \otimes S_{2}$ is unitarily equivalent to multiplication by $\zeta$ on the space $\int^{\oplus} \mathcal{N}_{\zeta} d \nu(\zeta)$

If $S_{1}$ and $S_{2}$ are Bergman operators or if they are cyclic subnormal operators, then Theorem 5.8 gives rather specific information. Let us examine the cyclic case first.

Let $S_{1}$ and $S_{2}$ be cyclic subnormal operators. From the general theory (see [7, pp. 146-147]) it can be assumed that each $S_{i}$ is multiplication by the independent variable on $P^{2}\left(\mu_{i}\right)$, the closure of the polynomials in $L^{2}\left(\mu_{i}\right)$. If $\mu=\mu_{1} \times \mu_{2}$, then it is not difficult to see that $P^{2}\left(\mu_{1}\right) \otimes P^{2}\left(\mu_{2}\right)=P^{2}(\mu)$, the $L^{2}(\mu)$ closure of the analytic polynomials in two variables. Thus $S_{1} \otimes S_{2}$ is multiplication by $z_{1} z_{2}$ on $P^{2}(\mu)$. 
5.9 Theorem. If $S_{i}$ is multiplication by $z$ on $P^{2}\left(\mu_{i}\right), i=1,2, \mu=\mu_{1} \times \mu_{2}$, $\mu_{1}$ and $\mu_{2}$ satisfy (5.5), and $\eta_{\zeta}$ is defined as in (5.6), then the minimal normal extension of $S_{1} \otimes S_{2}$ is unitarily equivalent to multiplication by $z_{1} z_{2}$ on

$$
\mathscr{N}=\left\{f \in L^{2}(\mu): f(z, \zeta / z) \in R_{0}^{2}\left(\eta_{\zeta}\right) \text { a.e. }[\nu]\right\},
$$

where $R_{0}^{2}\left(\eta_{\zeta}\right)$ is the closure in $L^{2}\left(\eta_{\zeta}\right)$ of the rational functions whose only pole is at 0.

Proof. If $p(z, w)$ is a polynomial, then for $\zeta \neq 0, p(z, \zeta / z)$ is a rational function whose only pole is at 0 . From (5.7), we see that $\mathscr{N}_{\zeta}=R_{0}^{2}\left(\eta_{\zeta}\right)$ for $\zeta \neq 0$. The theorem is now immediate from Theorem 5.8.

Once again employing direct integrals, the preceding theorem says that the minimal normal extension of $S_{1} \otimes S_{2}$ is unitarily equivalent to multiplication by $\zeta$ on $\int^{\oplus} R_{0}^{2}\left(\eta_{\zeta}\right) d \nu(\zeta)$.

5.10 Corollary. If $S_{1}$ and $S_{2}$ are cyclic subnormal operators, then the minimal normal extension of $S_{1} \otimes S_{2}$ is $N_{1} \otimes N_{2}$ if and only if $\operatorname{ker} N_{1}=\operatorname{ker} N_{2}=(0)$ and $R_{0}^{2}\left(\eta_{\zeta}\right)=L^{2}\left(\eta_{\zeta}\right)$ a.e. $[\nu]$.

Now let $S_{i}$ be the Bergman operator, multiplication by $z$ on $L_{a}^{2}\left(G_{i}\right)$ for some bounded open subset $G_{i}$ of $\mathbb{C}$. Theorem 5.8 applies to give the minimal normal extension of $S_{1} \otimes S_{2}$. However in this case The Coarea Formula can be used to obtain an explicit formula for the measure $\eta_{\zeta}$ defined in (5.7).

For $\zeta \neq 0$, note that the set $Y_{\zeta}=\left\{z \in G_{1}: \zeta / z \in G_{2}\right\}$ is an open subset of $G_{1}$ and $0 \notin \mathrm{cl} Y_{\zeta}$. If $G=G_{1} \times G_{2}$ and $\phi\left(z_{1}, z_{2}\right)=z_{1} z_{2}$, Lemma 4.12 implies that if $H_{1}$ is 1-dimensional Hausdorff measure on $\phi^{-1}(\zeta)$, then for $h$ in $L^{1}\left(H_{1}\right)$,

$$
\int_{\phi^{-1}(\zeta)} h d H_{1}=\int_{Y_{\zeta}} h(z, \zeta / z)|\zeta|^{-2}\left[|\zeta|^{2}+|z|^{4}\right] d \mathscr{A}_{1} .
$$

Thus

$$
d \eta_{\zeta}(z)=|\zeta|^{-2}\left[|\zeta|^{2}+|z|^{4}\right] d \mathscr{A}_{1}(z) \mid Y_{\zeta} .
$$

Each function in $L_{a}^{2}\left(G_{1} \times G_{2}\right)$ is the kernel of a Hilbert-Schmidt integral operator from $L_{a}^{2}\left(G_{1}\right)$ into $L_{a}^{2}\left(G_{2}\right)$. By using standard representations of such operators, we can conclude that $L_{a}^{2}\left(G_{1} \times G_{2}\right)=L_{a}^{2}\left(G_{1}\right) \otimes L_{a}^{2}\left(G_{2}\right)$. Combining this with Theorem 5.8 gives the following.

5.12 Theorem. Let $G_{1}$ and $G_{2}$ be bounded open subsets of $\mathbb{C}$ and for $\zeta \neq 0$, let $Y_{\zeta}=\left\{z \in G_{1}: \zeta / z \in G_{2}\right\}$. If $\eta_{\zeta}$ is the measure defined in (5.11) and $\mathscr{N}_{\zeta}=\bigvee\left\{h(z, \zeta / z): h \in L_{a}^{2}\left(G_{1} \times G_{2}\right)\right\}$, then the minimal normal extension of $S_{1} \otimes S_{2}$ is multiplication by $z_{1} z_{2}$ on

$$
\mathscr{N}=\left\{f \in L^{2}\left(G_{1} \times G_{2}\right): \text { for }\left[\mathscr{A}_{1}\right] \text { a.e. } \zeta, f(z, \zeta / z) \in \mathscr{N}_{\zeta}\right\} .
$$


Note from (5.11) that because $0 \notin \mathrm{cl} Y_{\zeta}, \eta_{\zeta}$ and $\mathscr{A}_{1} \mid Y_{\zeta}$ are boundedly mutually absolutely continuous measures. Thus for each $\zeta \neq 0, \mathscr{N}_{\zeta}$ is a closed subspace of $L_{a}^{2}\left(Y_{\zeta}\right)$. It seems possible that $\mathscr{N}_{\zeta} \neq L_{a}^{2}\left(Y_{\zeta}\right)$. When equality occurs a further improvement is possible.

As an illustration, consider the tensor product of the Bergman shift with itself. Here $S=$ multiplication by $z$ on $L_{a}^{2}(\mathbb{D})$ and $S \otimes S=$ multiplication by $z_{1} z_{2}$ on $L_{a}^{2}\left(\mathbb{D}^{2}\right)=L_{a}^{2}(\mathbb{D}) \otimes L_{a}^{2}(\mathbb{D})$.

5.13 Theorem. For $0<|\zeta|<1$, let $Y_{\zeta}$ be the annulus $\{z:|\zeta|<|z|<1\}$. If $S$ is the Bergman shift, then the minimal normal extension of the tensor product of $S$ with itself is multiplication by $z_{1} z_{2}$ on the space

$$
\left\{f \in L^{2}\left(\mathbb{D}^{2}\right): z \rightarrow f(z, \zeta / z) \in L_{a}^{2}\left(Y_{\zeta}\right) \text { for }\left[\mathscr{A}_{1}\right] \text { a.e. } \zeta\right\} .
$$

Proof. From Theorem 5.9 we know that the minimal normal extension of $S \otimes$ $S$ is multiplication by $z_{1} z_{2}$ on the space $\mathscr{H}=\left\{f \in L^{2}\left(\mathbb{D}^{2}\right): f(z, \zeta / z) \in\right.$ $R_{0}^{2}\left(\eta_{\zeta}\right)\left[\mathscr{A}_{1}\right]$ a.e. $\}$, where $\eta_{\zeta}$ is the measure defined on $Y_{\zeta}$ by $(5.11)$. As observed before, $\eta_{\zeta}$ and $\mathscr{A}_{1} \mid Y_{\zeta}$ are boundedly mutually absolutely continuous. Thus $R_{0}^{2}\left(\eta_{\zeta}\right)=L_{a}^{2}\left(Y_{\zeta}\right)$ for $\zeta \neq 0$.

If $\gamma$ is a smooth Jordan curve in $\mathbb{C}$, let $P^{2}(\gamma)$ denote the closure of the polynomials in $L^{2}(\gamma) \equiv$ the $L^{2}$ space of arc length measure. Also recall that if $G$ is a bounded Carathéodory region, the polynomials are dense in $L_{a}^{2}(G)$ [7, p. 186]. This paper concludes with an application of Theorem 5.9 to the cyclic subnormal operators defined by multiplication by $z$ on the spaces $P^{2}(\gamma)$ and $L_{a}^{2}(G)$.

5.14 Theorem. Let $\gamma$ be a smooth Jordan curve and let $G$ be a bounded Carathédory region. Let $S$ be multiplication by $z$ on $P^{2}(\gamma)$ and let $T$ be multiplication by $z$ on the Bergman space $L_{a}^{2}(G)$, with minimal normal extensions $N$ and $M$, respectively. If 0 lies either on or inside $\gamma$ or if for every nonzero $\zeta$ in $\mathbb{C}$ the curve $\zeta / \gamma$ is not contained in $\operatorname{cl} G$, then $N \otimes M$ is the minimal normal extension of $S \otimes T$. Conversely, if $N \otimes M$ is the minimal normal extension of $S \otimes T$, then either 0 lies on or inside $\gamma$ or for every nonzero $\zeta$ in $\mathbb{C}$, the curve $\zeta / \gamma$ is not contained in $G$.

Proof. Half the theorem can be proved immediately. Suppose that for every nonzero $\zeta$ the curve $\zeta / \gamma$ is not contained in $\operatorname{cl} G$. Hence $Y_{\zeta}=\{z \in \gamma: \zeta / z \in$ cl $G\}$ is a proper compact subset of $\gamma$. By Lavrentiev's Theorem $P\left(Y_{\zeta}\right)=C\left(Y_{\zeta}\right)$ and so $\mathscr{N}_{\zeta}$ (as defined in (5.7)) $=L^{2}\left(\eta_{\zeta}\right)$. Hence $N \otimes M$ is the minimal normal extension of $S \otimes T$ by Theorem 5.8. Now suppose that $0 \in \operatorname{cl}($ ins $\gamma$ ). If $0 \in \gamma$ and $\zeta \neq 0$, then $Y_{\zeta}$ is a compact subset of $\gamma$ that does not contain 0 and hence must be proper. Again Lavrentiev's Theorem implies $P\left(Y_{\zeta}\right)=C\left(Y_{\zeta}\right)$ and so $\mathscr{N}_{\zeta}=L^{2}\left(\eta_{\zeta}\right)$ for nonzero $\zeta$. Since area measure has no mass at 0 , Corollary 3.6 implies that $N_{1} \otimes N_{2}$ is the minimal normal extension of $S_{1} \otimes S_{2}$. If $0 \in$ ins $\gamma$, then the result follows by Theorem 5.3. 
To begin the proof of the converse, let $\gamma:[0,1] \rightarrow \mathbb{C}$ with $\gamma=u+i v$. Define $\phi:[0,1] \times G \rightarrow \mathbb{C}$ by $\phi(t, z)=\gamma(t) z$. Hence,

$$
\begin{aligned}
\phi(t, z) & =\phi(t, x, y)=(x+i y)(u(t)+i v(t)) \\
& =(x u-y v)+i(y u+x v) .
\end{aligned}
$$

Let $U(t, x, y)=x u(t)-y v(t)$ and $V(t, x, y)=y u(t)-x v(t)$ and calculate the Jacobian of $\phi$,

$$
|J \phi|^{2}=\left|\begin{array}{ll}
U_{t} & U_{y} \\
V_{t} & V_{y}
\end{array}\right|^{2}+\left|\begin{array}{ll}
U_{t} & U_{x} \\
V_{t} & V_{x}
\end{array}\right|^{2}+\left|\begin{array}{ll}
U_{x} & U_{y} \\
V_{x} & V_{y}
\end{array}\right|^{2} .
$$

Performing the necessary calculations we obtain

$$
|J \phi|^{2}=|\gamma|^{4}+|z|^{2}|\gamma|^{2}\left|\gamma^{\prime}\right|^{2}
$$

Now fix $\zeta=\alpha+i \beta \neq 0$. So $\phi^{-1}(\zeta)$ is a portion of the curve $\Phi_{\zeta}(t) \equiv$ $(t, \zeta / \gamma(t))$. Changing to real coordinates we get that $\phi^{-1}(\zeta)$ is a portion of the curve

$$
\Phi_{\zeta}(t)=\left(t,[\alpha u(t)+\beta v(t)]|\gamma(t)|^{-2},[\beta u(t)-\alpha v(t)]|\gamma(t)|^{-2}\right) \text {. }
$$

Performing the necessary calculations we obtain

$$
\left|\Phi_{\zeta}^{\prime}(t)\right|^{2}=\left[|\gamma(t)|^{4}+|\zeta|^{2}\left|\gamma^{\prime}(t)\right|^{2}\right]|\gamma(t)|^{-4} .
$$

If $I_{\zeta}=\{t \in[0,1]: \zeta / \gamma(t) \in G\}, L=\left\{\zeta \in \mathbb{C}: \phi^{-1}(\zeta) \neq \varnothing\right\}$, and $H_{\zeta}$ denotes 1-dimensional Hausdorff measure on $\phi^{-1}(\zeta)$, then The Coarea Formula gives that for $g$ in $L^{1}(\mu)$,

$$
\begin{aligned}
\int g d \mu & =\int_{L}\left[\int_{\phi^{-1}(\zeta)}|J \phi|^{-1} g d H_{\zeta}\right] d \mathscr{A}_{1}(\zeta) \\
& =\int_{L}\left[\int_{I_{\zeta}}\left|(J \phi)\left(\Phi_{\zeta}(t)\right)\right|^{-1} g\left(\Phi_{\zeta}(t)\right)\left|\Phi_{\zeta}^{\prime}(t)\right| d t\right] d \mathscr{A}_{1}(\zeta)
\end{aligned}
$$

After simplifying we have

$$
\int g d \mu=\int_{L}\left[\int_{I_{\zeta}}|\gamma(t)|^{-2} g\left(\Phi_{\zeta}(t)\right) d t\right] d \mathscr{A}_{1}(\zeta) .
$$

By the uniqueness of the disintegration of measures, the first equation in (5.15) is almost the disintegration of $\mu$ with respect to the function $\phi$. Specifically, if $\Lambda(\zeta)$ is the normalizing constant

$$
\Lambda(\zeta)=\int_{\phi^{-1}(\zeta)}|J \phi|^{-1} d H_{\zeta}
$$

then $d \nu(\zeta)=\Lambda(\zeta) d \mathscr{A}_{1}(\zeta)$ and $d \lambda_{\zeta}=\Lambda(\zeta)^{-1}|J \phi|^{-1} d H_{\zeta}$ gives the disintegration of $\mu$ with respect to the function $\phi$. If for any Borel set $E$ contained in 
$Y_{\zeta}$ we denote by $E_{\zeta}$ the set $\{(z, \zeta / z): z \in E\}$, then

$$
\begin{aligned}
\eta_{\zeta}(E) & =\lambda_{\zeta}\left(E_{\zeta}\right)=\Lambda(\zeta)^{-1} \int_{E_{\zeta}}|J \phi|^{-1} d H_{\zeta} \\
& =\Lambda(\zeta)^{-1} \int_{\gamma^{-1}(E)}\left|(J \phi)\left(\Phi_{\zeta}(t)\right)\right|^{-1}\left|\Phi_{\zeta}^{\prime}(t)\right| d t \\
& =\Lambda(\zeta)^{-1} \int_{\gamma^{-1}(E)}|\gamma(t)|^{-2} d t
\end{aligned}
$$

Multiplying and dividing by $\left|\gamma^{\prime}(t)\right|$ gives

$$
\eta_{\zeta}(E)=\Lambda(\zeta)^{-1} \int_{\gamma^{-1}(E)}\left[|\gamma(t)|^{2}\left|\gamma^{\prime}(t)\right|\right]^{-1}\left|\gamma^{\prime}(t)\right| d t .
$$

To complete the proof of the theorem, assume that the conditions are not satisfied. That is, assume that $0 \notin \operatorname{cl}($ ins $\gamma)$ and there is a $\zeta_{0}$ such that $\zeta_{0} / \gamma \subseteq$ $G$. Since $G$ is open, there is a $\delta>0$ such that $\zeta / \gamma \subseteq G$ for $\left|\zeta-\zeta_{0}\right|<\delta$. Hence $Y_{\zeta}=\gamma$ for $\left|\zeta-\zeta_{0}\right|<\delta$. Since $0 \notin \gamma$ and $\gamma$ is a smooth curve, there are positive constants $m$ and $M$ such that $m \leq|\gamma(t)|^{2}\left|\gamma^{\prime}(t)\right| \leq M$. Thus from (5.16), $\eta_{\zeta}$ and arc length measure on $\gamma$ are boundedly mutually absolutely continuous for $\left|\zeta-\zeta_{0}\right|<\delta$. Since $0 \notin$ ins $\gamma$, it follows that $R_{0}^{2}\left(\eta_{\zeta}\right) \neq L^{2}\left(\eta_{\zeta}\right)$ for $\left|\zeta-\zeta_{0}\right|<\delta$. Since this set of $\zeta$ has positive area, Corollary 5.10 implies that $N_{1} \otimes N_{2}$ is not the minimal normal extension of $S_{1} \otimes S_{2}$.

\section{BIBLIOGRAPHY}

1. M. B. Abrahamse and T. L. Kriete, The spectral multiplicity of a multiplication operator, Indiana Univ. Math. J. 22 (1973), 845-857.

2. W. B. Arveson, An invitation to $C^{*}$-algebra, Springer-Verlag, New York, 1976.

3. A. Athavale, Holomorphic kernels and commuting operators, Trans. Amer. Math. Soc. 304 (1987), 101-110.

4. E. Bishop, A generalization of the Stone-Weierstrass Theorem, Pacific J. Math. 11 (1961), 777-783.

5. N. Bourbaki, Éléments de mathématique, Livre VI, Intégration, Chapter 6, Intégration vectorielle, Hermann, Paris, 1959.

6. J. Chaumat, Adherence faible étoile d'algèbres de fractions rationelles, Publ. Math. Orsay 147 (1975).

7. J. B. Conway, Subnormal operators, Pitman, London, 1981.

8. _ A course in Functional analysis, Springer-Verlag, New York, 1985.

9. - The minimal normal extension of a function of a subnormal operator, Analysis at Urbana II, Proc. Special Year in Modern Analysis at Univ. of Illinois, 1986-87, Cambridge Univ. Press, Cambridge, 1989, pp. 128-140.

10. __ Towards a functional calculus for subnormal tuples: The minimal normal extension and approximation in several complex variables, Proc. Sympos. Pure Math., vol. 51, Part 2, Amer. Math. Soc., Providence, R. I., 1990, pp. 105-112.

11. J. B. Conway and R. F. Olin, A functional calculus for subnormal operators. II, Mem. Amer. Math. Soc. No. 184 (1977).

12. R. E. Curto, Applications of several complex variables to multiparameter spectral theory, Surveys of Some Recent Results in Operator Theory, vol. 2, Longman, London, 1988. 
13. 83 (1981), 730-734.

14. J. Dudziak, Spectral mapping theorems for subnormal operations, J. Funct. Anal. 56 (1984), 360-387.

15. _ The minimal normal extension problem for subnormal operators, J. Funct. Anal. 65 (1986), 314-338.

16. __ On a function-theoretic weak-star density problem connected with subnormal operators, Proc. Amer. Math. Soc. 107 (1989), 679-686.

17. T. W. Gamelin, Uniform algebras, Prentice-Hall, Englewood Cliffs, N.J., 1969.

18. T. W. Gamelin, P. Russo, and J. E. Thomson, A Stone-Weierstrass Theorem for weak-star approximation by rational functions, J. Funct. Annl. 87 (1989), 170-176.

19. I. Glicksberg, Measures orthogonal to algebras and sets of antisymmetry, Trans. Amer. Math. Soc. 105 (1962), 415-435.

20. T. Ito, On the commutative family of subnormal operators, J. Fac. Sci. Hokkaido Univ. 14 (1958), 1-15.

21. S. G. Krantz, Function theory of several complex variables, Wiley, New York, 1982.

22. A. Lubin, Weighted shifts and products of subnormal operators, Indiana Univ. Math. J. 26 (1977), 838-845.

23. F. Morgan, Geometric measure theory, Academic Press, Boston, Mass., 1988.

24. R. F. Olin, Functional relationships between a subnormal operator and its minimal normal extension, Pacific J. Math. 63 (1976), 221-229.

25. M. Putinar, Spectral inclusion for subnormal n-tuples, Proc. Amer. Math. Soc. 90 (1984), 405-406.

26. W. Rudin, Function theory in polydiscs, Benjamin, New York, 1969.

27. _ Function theory in the ball of $\mathbb{C}^{n}$, Springer-Verlag, New York, 1980.

28. R. Saerens, Interpolation theory in $\mathbb{C}^{n}:$ A survey, Complex Analysis (S. G. Krantz, Ed.), Lecture Notes in Math., vol. 1268, Springer-Verlag, Berlin, 1987.

29. D. Sarason, Weak-star density of polynomials, J. Reine Angew. Math. 252 (1972), 1-15.

30. J. L. Taylor, The analytic functional calculus for several commuting operators, Acta Math. 125 (1970), 1-30.

31. K. Yan, Invariant subspaces for joint subnormal systems (preprint).

Department of Mathematics, University of Tennessee, Knoxville, Tennessee 379961300 